\title{
A simplification problem in manifold theory
}

\author{
Jean-Claude HAUSMANN and Bjørn JAHREN
}

March 172018

\begin{abstract}
Two smooth manifolds $\mathrm{M}$ and $\mathrm{N}$ are called $\mathbb{R}$-diffeomorphic if $M \times \mathbb{R}$ is diffeomorphic to $N \times \mathbb{R}$. We consider the following simplification problem: does $\mathbb{R}$-diffeomorphism imply diffeomorphism or homeomorphism? For compact manifolds, analysis of this problem relies on some of the main achievements of the theory of manifolds, in particular the $\mathrm{h}$ - and scobordism theorems in high dimensions and the spectacular more recent classification results in dimensions 3 and 4 . This paper presents what is currently known about the subject as well as some new results about classifications of $\mathbb{R}$-diffeomorphisms.
\end{abstract}

\section{Contents}

\begin{tabular}{lll}
\hline & Introduction & 1
\end{tabular}

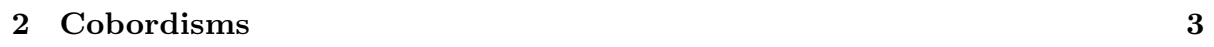

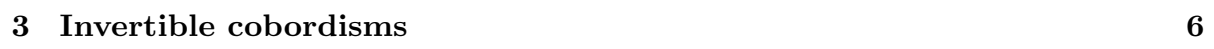

$\begin{array}{lll}4 & \text { The case } n \geq 5 & 15\end{array}$

$\begin{array}{lll}5 & \text { The case } n=4 & 19\end{array}$

\begin{tabular}{lll}
\hline 6 & The case $n \leq 3$ & 22
\end{tabular}

\begin{tabular}{|ll|}
\hline 7 & Classifications of $\mathbb{R}$-diffeomorphisms \\
\hline
\end{tabular}

\begin{tabular}{|lll}
\hline & Miscellaneous & 31
\end{tabular}

\section{Introduction}

Let $X$ and $Y$ be smooth manifolds. We write $Y \approx_{\text {diff }} X$ when $Y$ is diffeomorphic to $Y$ and $Y \approx_{\text {top }} X$ when $Y$ is homeomorphic to $Y$. Given a manifold $P, Y$ and $X$ are called $P$-diffeomorphic (notation: $X \approx \approx_{P \text {-diff }} Y$ ) if there exists a diffeomorphism $f: Y \times P \rightarrow X \times P$, and such an $f$ is called a $P$-diffeomorphism. Consider the following simplification problem. 
The P-Simplification Problem. $\quad$ For smooth closed manifolds, does Pdiffeomorphism imply diffeomorphism, or homeomorphism?

The first part of this paper is a survey on what is currently known about the $\mathbb{R}$-simplification problem (other cases are briefly discussed in Section 8). This quite natural question, expressed in very elementary terms, happens to be closely related to the theory of invertible cobordisms (see e. g. [59, 28, and Proposition 3.3. As advertisement, here are some samples of the main results of the theory.

Theorem A. Let $M$ and $N$ be smooth closed manifolds of dimension n. Suppose that $M$ is simply connected. Then

(i) $N \approx_{\mathbb{R} \text {-diff }} M \Longrightarrow N \approx_{\text {top }} M$.

(ii) $N \approx_{\mathbb{R} \text {-diff }} M \Longrightarrow N \approx_{\text {diff }} M$ if $n \neq 4$.

The simplicity of the statement of Theorem A, with almost no dimension restriction, contrasts with the variety of techniques involved in the proof. Actually, Theorem A concentrates a good deal of important developments in differential topology during the 20th century (see 8.2).

When $M$ is not simply connected, part (i) is false in general, as first discovered by J. Milnor in 1961 [48, with $M=L(7,1) \times S^{4}$ and $N=L(7,2) \times S^{4}$. Using a recent result of Jahren-Kwasik [29, Theorem 1.2], we now know that Part (i) of Theorem A is, in general, "infinitely false", i.e. there are manifolds having countably many homeomorphism classes within their $\mathbb{R}$-diffeomorphism class (see Example 4.5.(5)).

In dimension 4, part (ii) of Theorem $\mathrm{A}$ is infinitely false in general, even when $M$ is simply connected. Indeed, for $M=\mathbb{C} P^{2} \sharp k \overline{\mathbb{C} P^{2}}(k \geq 6)$, there exist countably many diffeomorphism classes of manifolds homeomorphic to $M$ [13. These provide counterexamples of part (ii) of Theorem A, thanks to the following result (probably known by specialists).

Theorem B. Let $M$ and $N$ be smooth closed manifolds of dimension 4 which are homeomorphic. Suppose that $H_{1}\left(M, \mathbb{Z}_{2}\right)=0$. Then $N \approx_{\mathbb{R} \text {-diff }} M$.

For $M$ and $N$ as in Theorem B but simply connected, $N \approx_{\text {top }} M$ if and only if $M$ and $N$ have the same homotopy type [14, $\S 10.1]$. In particular, exotic spheres, if any, would all be $\mathbb{R}$-diffeomorphic.

The hypothesis of simple connectivity in Theorem A is not necessary in low dimensions. The following result is classical for $n \leq 2$, follows for $n=3$ from a result of Turaev 61] together with the geometrization theorem.

Theorem C. Let $M$ and $N$ be two closed manifolds of dimension $n \leq 3$, which are orientable if $n=3$. Then $N \approx_{\mathbb{R} \text {-diff }} M$ if and only if $N \approx_{\text {diff }} M$.

Theorem $\mathrm{C}$ is currently unknown for non-orientable 3-manifolds (see Remark 6.2).

Proofs of theorems A, B and C are given in Sections 4-6 (with more general hypotheses for Theorem A), after important preliminaries in Sections 2-3. Of 
particular importance for the simplification problem are the so-called inertial invertible cobordisms, characterized by the property that the two ends are diffeomorphic (homeomorphic). Section 4 also includes some new results in this area (notably Proposition 4.7).

In the last part of this paper (Section 7), we present new results on classification of $\mathbb{R}$-diffeomorphisms under several equivalence relations. For instance, a diffeomorphism $f: N \times \mathbb{R} \rightarrow M \times \mathbb{R}$ is called decomposable if there exists a diffeomorphism $\varphi: N \rightarrow M$ such that $f$ is isotopic to $\varphi \times \pm \operatorname{id}_{\mathbb{R}}$. Fix a manifold $M$ and consider pairs $(N, f)$ where $N$ is a smooth closed manifold and $f: N \times \mathbb{R} \rightarrow M \times \mathbb{R}$ is a diffeomorphism. Two such pairs $(N, f)$ and $(\hat{N}, \hat{f})$ are equivalent if $f^{-1} \circ \hat{f}$ is decomposable. The set of equivalence classes is denoted by $\mathcal{D}(M)$. We compute this set in all dimensions in terms of invertible cobordisms. As a consequence, in high dimensions we get the following result.

Theorem D. Let $M$ be a closed connected smooth manifold of dimension $n \geq 5$. Then $\mathcal{D}(M)$ is in bijection with the Whitehead group $\mathrm{Wh}\left(\pi_{1} M\right)$.

Corollary E. Let $M$ be a closed connected smooth manifold of dimension $n \geq 5$. The following assertions are equivalent.

(i) $\mathrm{Wh}\left(\pi_{1} M\right)=0$.

(ii) Any diffeomorphism $f: N \times \mathbb{R} \rightarrow M \times \mathbb{R}$ is decomposable.

Theorem D is actually a consequence of a more categorical statement (Theorem 7.1), which is of independent interest.

We also consider a quotient $\mathcal{D}_{c}(M)$ of $\mathcal{D}(M)$ where isotopy is replaced by concordance. Interesting examples are produced to discuss the principle of concordance implies isotopy for $\mathbb{R}$-diffeomorphisms.

Acknowledgments: The first author thanks Pierre de la Harpe and Claude Weber for useful discussions. The second author thanks Sławomir Kwasik for numerous enlightening discussion about material related to this paper. We also thank Matthias Kreck and Raphael Torres for useful comments.

\section{Cobordisms}

2.1. Preliminaries. Throughout this paper, we work in the smooth category $\mathcal{C}^{\infty}$ of smooth manifolds, (possibly with corners: see below) and smooth maps. Our manifolds are not necessarily orientable.

If $X$ is a manifold and $r \in \mathbb{R}$, the formula $j_{X}^{r}(x)=(x, r)$ define a diffeomorphism $j_{X}^{r}: X \rightarrow X \times\{r\}$ or an embedding $j_{X}^{r}: X \rightarrow X \times \mathbb{R}$, depending on the context.

Let $(X, Y)$ and $\left(X^{\prime}, Y^{\prime}\right)$ be manifolds, with an identification (diffeomorphism) $\imath: Y \stackrel{\approx}{\longrightarrow} Y^{\prime}$, usually obvious in the context (as $j_{Y}^{r}$ above). A map $f: X \rightarrow X^{\prime}$ is called relative $Y$ (notation: rel $Y$ ) if the restriction of $f$ to $Y$ coincides with the identification $\imath$. Often, $Y=\partial X$ and $Y^{\prime}=\partial X^{\prime}$, in which case we say relative boundary (notation: rel $\partial$ ). 
2.2. The cobordism category. A triad is a triple $(W, M, N)$ of compact smooth manifolds such that $\partial W=(M \amalg N) \cup X$ with $X \approx_{\text {diff }} \partial M \times I$. Most often $\partial M$ is empty, in which case $\partial W=M \amalg N$. Otherwise, $W$ is actually a manifold with corners along $\partial M$ and $\partial N$, modeled locally on the subset $\left\{\left(x_{1}, \ldots, x_{n}\right) \mid x_{1} \geq 0, x_{2} \geq 0\right\}$ of $\mathbb{R}^{n}$. Smooth maps are then always required to preserve the stratification coming from this local structure (for a precise exposition of the smooth category with corners, see the appendix of [4]).

Let us fix the manifolds $M$ and $N$ (one or both of them could be empty). A cobordism from $M$ to $N$ is a triple $\left(W, j_{M}, j_{N}\right)$, where $W$ is a compact smooth manifold and $j_{M}: M \rightarrow \partial W, j_{N}: N \rightarrow \partial W$ are embeddings such that $\left(W, j_{M}(M), j_{N}(N)\right)$ is a triad. If $M$ and $N$ have nonempty boundaries, $\left(W, j_{M}, j_{N}\right)$ will sometimes be called a relative cobordism.

By a slight abuse of notation we will also let $j_{M}$ denote the embedding $j_{M}$ considered as a map into $W$.

Two cobordisms $\left(W, j_{M}, j_{N}\right)$ and $\left(W^{\prime}, j_{M}^{\prime}, j_{N}^{\prime}\right)$ are equivalent if there is a diffeomorphism $h: W \rightarrow W^{\prime}$ such that $j_{M} \circ h=j_{M}^{\prime}$ and $j_{N} \circ h=j_{N}^{\prime}$. The set of equivalence classes of cobordisms from $M$ to $N$ is denoted by $\operatorname{Cob}(M, N)$. The equivalence class of $\left(W, j_{M}, j_{N}\right)$ is denoted by $\left[W, j_{M}, j_{N}\right]$.

A triad $(W, M, N)$ determines an obvious cobordism, $\left(W, \imath_{M}, \imath_{N}\right)$, and its equivalence class in $\operatorname{Cob}(M, N)$ will also be denoted by $[W, M, N]$. Note that $[W, M, N]=\left[W^{\prime}, M, N\right]$ if and only if $W \approx_{\text {diff }} W^{\prime}(\operatorname{rel} M \cup N)$. We shall make no distinction between a triad and the cobordism it determines and often write "a cobordism $(W, M, N)$ " instead of "a triad $(W, M, N)$ ". A triad of the form $(M \times I, M \times 0, M \times 1)=\left(M \times I, j_{M}^{0}, j_{N}^{1}\right)$ (using the notations $j_{X}^{r}$ from 2.1) will be called a trivial cobordism.

We now define a composition

$$
\operatorname{Cob}(M, N) \times \operatorname{Cob}(N, P) \stackrel{\circ}{\longrightarrow} \operatorname{Cob}(M, P) .
$$

Let $c \in \operatorname{Cob}(M, N)$ and $c^{\prime} \in \operatorname{Cob}(N, P)$, represented by cobordisms $\left(W, j_{M}, j_{N}\right)$ and $\left(W^{\prime}, j_{N}^{\prime}, j_{P}^{\prime}\right)$. The topological manifold $W \cup_{j_{N}^{\prime} \circ j_{N}^{-1}} W^{\prime}$ admits a smooth structure compatible with those on $W$ and $W^{\prime}$ [49, Theorem 1.4]. Such a smooth structure is unique up to diffeomorphism relative boundary (see also [26. Chapter $8, \S 2])$. Choosing one of these smooth structures gives rise to a smooth manifold $W \circ W^{\prime}$, and $\left(W \circ W^{\prime}, j_{M}, j_{P}^{\prime}\right)$ represents a well-defined class $c_{\circ} c^{\prime} \in \operatorname{Cob}(M, P)$. With this composition, one gets a category Cob whose objects are closed smooth manifolds and whose set of morphisms from $M$ to $N$ is $\operatorname{Cob}(M, N)$. The identity at the object $M$ is represented by the trivial cobordism:

$$
\mathbf{1}_{M}=[M \times I, M \times 0, M \times 1]=\left[M \times I, j_{M}^{0}, j_{N}^{1}\right] .
$$

Note that, by construction, the composition $\mathbf{1}_{M} \circ\left(W, j_{M}, j_{N}\right) \circ \mathbf{1}_{N}$ has the form of a triad $\left(W^{\prime}, M, N\right)$, where we identify $M$ and $N$ with $M \times 0$ and $N \times 1$. In other words: up to equivalence, cobordisms can always be represented by triads. This will sometimes be exploited in proofs, in order to simplify notation. But in general it is helpful to have the extra flexibility of the more general 
definition, as it makes it easier to keep track of how we identify $M$ and $N$ with submanifolds of $\partial W$. A trivial example is $\mathbf{1}_{M}$, which as cobordism goes from $M$ to itself, but in a triad the two ends can not be the same manifold. More examples are the definition of mapping cylinders and Lemma 2.4 below.

Our definition of the cobordism category is a condensed reformulation of 49, $\S 1$, with end-identifications going in reverse directions.

2.3. Duals and mapping cylinders. The order of $M$ and $N$ in $\left(W, j_{M}, j_{N}\right)$ reflects the categorical intuition that $W$ is a cobordism from $M$ to $N$. Reversing the order of $M$ and $N$, we obtain the dual cobordism $\left(\bar{W}, j_{N}, j_{M}\right)$, where $\bar{W}$ is just a copy of $W$. If the cobordism is given by a triad $(W, M, N)$, its dual is given by $(\bar{W}, N, M)$. The correspondence $[W] \rightarrow[\bar{W}]$ defines a functor $\mathrm{Cob} \rightarrow \mathrm{Cob}^{\text {op }}$ which is an isomorphism of categories.

Examples of cobordisms are given by mapping cylinders of diffeomorphisms. Let $f: M \rightarrow N$ be a diffeomorphism between smooth closed manifolds. The mapping cylinder $C_{f}$ of $f$ is defined by

$C_{f}=\{M \times[0,1)\} \cup\{N \times(0,1]\} /\{(x, t) \sim(f(x), t)$ for all $(x, t) \in M \times(0,1)\}$.

Note the obvious homeomorphism

$$
C_{f} \approx_{\text {top }}\{M \times I \cup N\} /\{(x, 1) \sim f(x)\} .
$$

The latter is the usual definition of the mapping cylinder valid for any continuous map $f$. But, when $f$ is a diffeomorphism, Definition (2.1) makes $C_{f}$ a smooth manifold with boundary $\partial C_{f}=M \times\{0\} \cup N \times\{1\}$. We thus get a cobordism $\left(C_{f}, j_{M}^{0}, j_{N}^{1}\right)$.

Lemma 2.4. For a diffeomorphism $f: M \rightarrow N$ between smooth closed manifolds, the equalities

$$
\left[C_{f}, j_{M}^{0}, j_{N}^{1}\right]=\left[M \times I, j_{M}^{0}, j_{M}^{1} \circ f^{-1}\right]=\left[N \times I, j_{N}^{0} \circ f, j_{N}^{1}\right]
$$

hold in $\operatorname{Cob}(M, N)$.

Proof. One checks that the correspondences

$$
\left\{\begin{aligned}
M \times[0,1) \ni(x, t) & \mapsto(x, t) \\
N \times(0,1] \ni(y, t) & \mapsto\left(f^{-1}(y), t\right) .
\end{aligned}\right.
$$

provide the first equality. The second one is obtained similarly.

Example 2.5. Let $f: M \rightarrow M$ be a self-diffeomorphism of a closed manifold $M$. Then $C_{f}$ is equivalent to $\mathbf{1}_{M}$ if and only if there is a diffeomorphism $F: M \times I \rightarrow M \times I$ such that $F(x, 0)=f(x)$ and $F(x, 1)=x$, i.e. $F$ is concordant to $\mathrm{id}_{M}$.

The proof of the following lemma is left to the reader (compare 49, Theorems 1.6]). 
Lemma 2.6. Let $M \stackrel{f}{\longrightarrow} N \stackrel{g}{\longrightarrow} P$ be diffeomorphisms between smooth manifolds. Then $\left[C_{g \circ f}\right]=\left[C_{f}\right] \circ\left[C_{g}\right]$.

Remark. The reason for the contravariant form of this identity is that we write composition of cobordisms "from left to right". This is the usual convention in cobordism categories, like path categories (e. g. fundamental groupoid) and topological field theories.

\section{Invertible cobordisms}

3.1. The category of invertible cobordisms. A cobordism $\left(W, j_{M}, j_{N}\right)$ is called invertible if $[W]$ is an invertible morphism in Cob, i. e. there is a cobordism $\left(W^{-1}, j_{N}, j_{M}\right)$ such that $[W] \circ\left[W^{-1}\right]=\mathbf{1}_{M}$ and $\left[W^{-1}\right] \circ[W]=\mathbf{1}_{N}$.

As usual, these conditions uniquely determine $\left[W^{-1}\right]$ if it exists. Two smooth manifolds are invertibly cobordant if there exists an invertible cobordism between them. Let $\operatorname{Cob}^{*}(M, N)$ be the subset of $\operatorname{Cob}(M, N)$ formed by invertible cobordisms. This defines a subcategory $\mathrm{Cob}^{*}$ of $\mathrm{Cob}$, with the same objects.

An example of invertible cobordism is given by the mapping cylinder $C_{f}$ of a diffeomorphism $f: N \rightarrow M$. Indeed, Lemma 2.6 together with Lemma 2.4 imply that $\left[C_{f}\right]^{-1}=\left[C_{f-1}\right]=\left[\overline{C_{f}}\right]$.

3.2. Invertible cobordisms and $\mathbb{R}$-diffeomorphisms. From now on until section 7 we will be mainly concerned with cobordisms between closed manifolds, unless explicitly stated. The main exceptions are the discussions of h-cobordism and Whitehead torsion in 3.10 and 3.12 and of concordance in 3.17 .

Here is one of the main results of this section.

Proposition 3.3. Let $M$ and $N$ be smooth closed manifolds. The following statements are equivalent.

(a) $N \approx_{\mathbb{R} \text {-diff }} M$

(b) $N$ and $M$ are invertibly cobordant.

(c) There is a diffeomorphism $\beta: N \times S^{1} \rightarrow M \times S^{1}$ such that the composed homomorphism

$$
\pi_{1}(N \times p t) \longrightarrow \pi_{1}\left(N \times S^{1}\right) \stackrel{\beta_{*}}{\longrightarrow} \pi_{1}\left(M \times S^{1}\right) \stackrel{\text { proj }}{\longrightarrow} \pi_{1}\left(S^{1}\right)
$$

is trivial.

(d) There is a diffeomorphism $\beta: N \times S^{1} \rightarrow M \times S^{1}$ such that the diagram

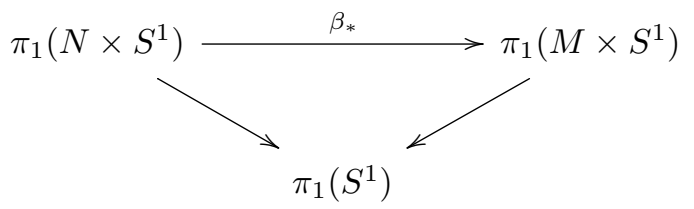


commutes, where the arrows to $\pi_{1}\left(S^{1}\right)$ are induced by the projections onto $S^{1}$

Remark 3.4. Conditions (c) or (d) are stronger than just $S^{1}$-diffeomorphism, since there are examples of closed manifolds $M$ and $N$ such that $M \approx_{S^{1} \text {-diff }} N$ but $\pi_{1}(N) \not \approx \pi_{1}(M)$ (see e.g. [8, p. 29], [10, Theorem 4.1] or [36, Theorem 2]). Some of these examples are in dimension 3 , so crossing with spheres provide examples in all dimensions greater than four.

We write a detailed proof of Proposition 3.3 introducing notations which will be useful in Section 7. Also, proving $(a) \Rightarrow(c)$ is delicate: Kervaire wrote a short argument at the end of 32 but, after publication, thought that his argument was incorrect. For a proof of $(b) \Rightarrow(c)$ using the deep s-cobordism theorem, when $\operatorname{dim} M \geq 4$, see Remark 3.16 .

Proof of Proposition 3.3. (a) implies (b). Let $f: N \times \mathbb{R} \rightarrow M \times \mathbb{R}$ be a diffeomorphism. Write $M_{u}=M \times\{u\}, N_{u}=N \times\{u\}$ and $N_{u}^{\prime}=f\left(N_{u}\right)$. We use the obvious diffeomorphisms $j_{M}^{u}: M \rightarrow M_{u}$ and $j_{N}^{u}: N \rightarrow N_{u}$ introduced in Section 2.1.

By compactness of $N$, there exists $r<u<s<v$ such that $N_{u}^{\prime} \subset M \times(r, s)$ and $M_{s} \subset f(N \times(u, v))$ (to get this order, one might have to precompose $f$ by the automorphism $(x, u) \mapsto(x,-u)$ of $N \times \mathbb{R})$. The region $A$ between $M_{r}$ and $N_{u}^{\prime}$ and the region $B$ between $N_{u}^{\prime}$ and $M_{s}$ produce equivalence classes of cobordisms

$$
\left[A, j_{M}^{r}, f \circ j_{N}^{u}\right] \in \operatorname{Cob}(M, N),\left[B, f \circ j_{N}^{u}, j_{M}^{s}\right] \in \operatorname{Cob}(N, M)
$$

obviously satisfying $[A] \circ[B]=\mathbf{1}_{M}$. One also has the class of cobordism

$$
\left[A^{\prime}, j_{M}^{s}, f \circ j_{N}^{v}\right] \in \operatorname{Cob}(M, N) \text {. }
$$

Using the diffeomorphism $f$, one proves that $[B] \circ\left[A^{\prime}\right]=\mathbf{1}_{N}$. This implies that $\left[A^{\prime}\right]=[A]$ and $[B]=[A]^{-1}$.

(b) implies (a) and (c). We first prove that (b) implies (a), using an argument of Stallings [59, $\S 2]$. Let $A$ be an invertible cobordism from $M$ to $N$, with inverse $B$. Let $A_{i}$ and $B_{i}$ be copies of $A$ and $B$ indexed by $i \in \mathbb{Z}$. Consider the manifold

$$
\begin{aligned}
W & =\cdots \circ\left(A_{i} \circ B_{i}\right) \circ\left(A_{i+1} \circ B_{i+1}\right) \circ \cdots \\
& =\cdots \circ\left(B_{i} \circ A_{i+1}\right) \circ\left(B_{i+1} \circ A_{i+2}\right) \circ \cdots
\end{aligned}
$$

Let $g_{i}: M \times[i, i+1] \rightarrow A_{i} \circ B_{i}$ be copies of some diffeomorphism relative boundary $g: M \times I \rightarrow A \circ B$. Then, $g_{M}=\bigcup_{i \in \mathbb{Z}} g_{i}$ is a diffeomorphism from $M \times \mathbb{R}$ onto $W$. The same may be done with the second decomposition of $W$. We thus get two diffeomorphisms $g_{M}: M \times \mathbb{R} \rightarrow W$ and $g_{N}: N \times \mathbb{R} \rightarrow W$, which proves (a).

We now prove (c). By conjugation by $g_{M}$, the automorphism $(x, t) \rightarrow(x, t+$ 1) of $M \times \mathbb{R}$ produces an automorphism $T$ of $W$, generating a free and proper $\mathbb{Z}$ action on $W$ and a diffeomorphism $\alpha: W / \mathbb{Z} \stackrel{\approx}{\longrightarrow} M \times S^{1}$. It is not clear whether 
the corresponding automorphism obtained via $g_{N}$ is conjugate to $T$. However, the manifold $Z_{i}=B_{i} \circ A_{i+1}$ is a fundamental domain for the $T$-action and the restriction of $T$ to $Z_{i}$ sends $Z_{i}$ onto $Z_{i+1}$ relative boundary. Therefore, we get a diffeomorphism

$$
\beta: N \times S^{1} \underset{\approx_{\text {diff }}}{\longrightarrow} N \times(I / \partial I) \underset{\approx_{\text {diff }}}{\longrightarrow} W / \mathbb{Z} \underset{\approx_{\text {diff }}}{\longrightarrow} M \times S^{1}
$$

The composed homomorphism 3.5 is trivial since the restriction of $\beta$ to $N \times p t$ factors through $M \times \mathbb{R}$.

(c) implies (d). Using the exact sequence

$$
1 \rightarrow \pi_{1}(N \times p t) \rightarrow \pi_{1}\left(N \times S^{1}\right) \stackrel{\text { proj }}{\longrightarrow} \pi_{1}\left(S^{1}\right) \rightarrow 1
$$

Condition (d) implies that $\operatorname{proj} \circ \beta_{*}$ factors through an endomorphism $\bar{\beta}_{*}$ of $\pi_{1}\left(S^{1}\right)$ which, being surjective, satisfies $\bar{\beta}_{*}(b)= \pm b$ (identifying $\pi_{1}\left(S^{1}\right)$ with $\mathbb{Z})$. The possible negative sign may be avoided by precomposing $\beta$ with the automorphism $(x, z) \mapsto(x, \bar{z})$ of $N \times S^{1}$.

(d) implies (a). Let $\beta: N \times S^{1} \rightarrow M \times S^{1}$ as in (d). Consider the pullback diagram

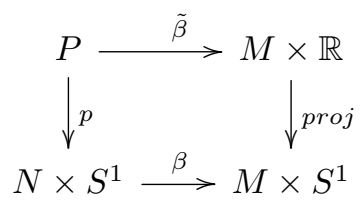

The map $\tilde{\beta}$ is a diffeomorphism, since so is $\beta$. The covering $p$ corresponds to the homomorphism $\operatorname{proj}_{\circ} \beta_{*}: \pi_{1}\left(N \times S^{1}\right) \rightarrow \pi_{1}\left(S^{1}\right)$. The latter is equal to $\operatorname{proj}: \pi_{1}\left(N \times S^{1}\right) \rightarrow \pi_{1}\left(S^{1}\right)$ by the commutativity of $(3.6)$, implying that $P \approx_{\text {diff }} N \times \mathbb{R}$.

Closely related to Proposition 3.3 is the following result.

Proposition 3.5. Let $\left(W, j_{M}, j_{N}\right)$ be a cobordism between closed, compact manifolds. The following five statements are equivalent:

(a) $W$ is invertible.

(b) $W-j_{N}(N) \approx_{\text {diff }} M \times[0, \infty) . \quad\left(b^{\prime}\right) W-j_{M}(M) \approx_{\operatorname{diff}} N \times(-\infty, 0]$.

(c) $W-\partial W \approx_{\text {diff }} M \times \mathbb{R} . \quad \quad\left(c^{\prime}\right) W-\partial W \approx_{\text {diff }} N \times \mathbb{R}$.

Proof. It clearly suffices to prove this for a triad $(W, M, N)$. We shall prove that $(a) \Rightarrow(b) \Rightarrow(c) \Rightarrow(a)$. The implication chain $(a) \Rightarrow\left(b^{\prime}\right) \Rightarrow\left(c^{\prime}\right) \Rightarrow(a)$ is obtained similarly. 
Suppose that $W$ admits an inverse $W^{-1}$. Let $W_{i}$ and $W_{i}^{-1}$ be copies of $W$ and $W^{-1}$, indexed by $i \in \mathbb{N}$. One has

$$
\begin{aligned}
W-N & \approx_{\text {diff }} \quad W \circ N \times[0, \infty) \\
& \approx_{\text {diff }} \quad W_{0} \circ W_{0}^{-1} \circ W_{1} \circ W_{1}^{-1} \circ W_{2} \circ \cdots \\
\approx_{\text {diff }} & M \times[0,1] \circ W_{1} \circ W_{1}^{-1} \circ W_{2} \circ \cdots \\
& \approx_{\text {diff }} \quad M \times[0, \infty)
\end{aligned}
$$

thus $(a) \Rightarrow(b)$.

As $(b) \Rightarrow(c)$ is obvious, it remains to prove $(c) \Rightarrow(a)$. For $1 \leq r \in \mathbb{N}$, let $W_{r}=(M \times[-r, 0]) \circ W \circ(N \times[0, r])$ and $V_{r}=M \times[-r, r]$. Let $f: W-\partial W \rightarrow$ $M \times \mathbb{R}$ be a diffeomorphism. As $W-\partial W \approx_{\text {diff }} \lim _{r \rightarrow \infty} W_{r}$ and $M \times \mathbb{R} \approx_{\text {diff }}$ $\lim _{r \rightarrow \infty} V_{r}$, there are $1 \leq r<s<t$ in $\mathbb{N}$ such that

$$
f\left(W_{0}\right) \subset V_{r} \subset f\left(W_{s}\right) \subset V_{t}
$$

none of these inclusions being an equality. As in the proof of Proposition 3.3. this provides classes $A, B, C, X, Y, Z$ in $\operatorname{Cob}(M, M)$ such that $\left[V_{r}\right]=A \circ\left[W_{0}\right] \circ X$, $\left[W_{s}\right]=B \circ\left[V_{r}\right] \circ Y$ and $\left[V_{t}\right]=C \circ\left[W_{s}\right] \circ Z$. Moreover, $B \circ A=[M \times[-s, 0]]=\mathbf{1}_{M}$ and $C \circ B=[M \times[-t,-s]]=\mathbf{1}_{M}$. Therefore, $B$ is invertible and $C=A=B^{-1}$. In the same way, $Y$ is invertible and $X=Z=Y^{-1}$. Therefore,

$$
[W]=\left[W_{s}\right]=B \circ\left[V_{r}\right] \circ Y=B \circ \mathbf{1}_{M} \circ Y=B \circ Y
$$

and thus $W$ is invertible.

3.6. The set $\mathcal{B}(M)$. In view of Proposition 3.3. the study of the simplification problem is related to the classification of invertible cobordisms. We fix a smooth closed connected manifold $M$ and consider invertible cobordisms starting from $M$. Two such cobordisms are regarded as equivalent if they are diffeomorphic relative to $M$. To be precise: $\left(W, j_{M}, j_{N}\right)$ is equivalent to $\left(W^{\prime}, j_{M}^{\prime}, j_{N}^{\prime}\right)$ if there is a diffeomorphism $f: W \approx_{\text {diff }} W^{\prime}$ such that $j_{M}^{\prime}=f j_{M}$. The equivalence class of a cobordism $\left(W, j_{M}, j_{N}\right)$ does not depend on $j_{N}$ and is denoted by $\left[W, j_{M}[\right.$, or just $[W[$. Let $\mathcal{B}(M)$ be the set of equivalence classes.

Example 3.7. Let $\left(W, j_{M}, j_{N}\right)$ be an invertible cobordism between closed manifolds $M$ and $N$. Then, $\left[W, j_{M}\left[=\left[M \times I, j_{M}^{0}[\right.\right.\right.$ in $\mathcal{B}(M)$ if and only if $[W]=\left[C_{f}\right]$ for some diffeomorphism $f: M \rightarrow N$. Indeed, the if part follows from Lemma 2.4. Conversely, let $F: M \times I \rightarrow W$ be a diffeomorphism rel $M \times 0$ and let $f: M \rightarrow N$ be the restriction of $F$ to $M \times 1$. Then $F^{-1} \circ j_{N}=j_{M}^{1} \circ f^{-1}$, which implies that $\left[W, j_{M}, j_{N}\right]=\left[M \times I, j_{M}^{0}, j_{M}^{1} \circ f^{-1}\right]$. The latter coincides with $\left[C_{f}\right]$ by Lemma 2.4 again.

For any closed manifold $N$, the correspondence $[W] \mapsto[W[$ gives a map $\tilde{\alpha}_{M, N}: \operatorname{Cob}^{*}(M, N) \rightarrow \mathcal{B}(M)$ that we shall now study (note that $\operatorname{Cob}^{*}(M, N)$ is empty if $N$ is not invertibly cobordant to $M)$. The group $\operatorname{Diff}(N)$ of selfdiffeomorphisms of $N$ acts on the right on $\operatorname{Cob}^{*}(M, N)$ by $\left[W, j_{M}, j_{N}\right] \varphi=$ $\left[W, j_{M}, j_{N} \circ \varphi\right]$. The map $\tilde{\alpha}_{M, N}$ is invariant for this action and then descends 
to a map $\alpha_{M, N}$ : $\operatorname{Cob}^{*}(M, N) / \operatorname{Diff}(N) \rightarrow \mathcal{B}(M)$. We claim that the latter is injective. Indeed, if $\tilde{\alpha}_{M, N}\left(\left[W, j_{M}, j_{N}\right]\right)=\tilde{\alpha}_{M, N}\left(\left[W^{\prime}, j_{M}^{\prime}, \hat{j}_{N}^{\prime}\right]\right)$, then there is a diffeomorphism $h: W \rightarrow W$ such that $h \circ j_{M}=j_{M}^{\prime}$ and thus

$$
\left[W, j_{M}, j_{N}\right]=\left[W^{\prime}, j_{M}^{\prime}, h_{\circ} j_{N}\right]=\left[W^{\prime}, j_{M}, j_{N}^{\prime}\right] k,
$$

where $k=\left(j_{N}^{\prime}\right)^{-1} h_{\circ} j_{N} \in \operatorname{Diff}(N)$.

Let $\mathcal{M}_{n}$ be the set of diffeomorphism classes of closed manifolds of dimension $n$. The correspondence $(W, M, N) \mapsto[N]$ defines a map

$$
e: \mathcal{B}(M) \rightarrow \mathcal{M}_{n} .
$$

Let $\mathcal{M}_{n}^{0}$ be a set of representatives of $\mathcal{M}_{n}$ (one manifold for each class).

Lemma 3.8. The map $\alpha=\amalg_{N \in \mathcal{M}_{n}^{0}} \alpha_{M, N}$ provides a bijection

$$
\coprod_{N \in \mathcal{M}_{n}^{0}} \operatorname{Cob}^{*}(M, N) / \operatorname{Diff}(N) \stackrel{\alpha}{\underset{\sim}{\longrightarrow}} \mathcal{B}(M) .
$$

The resulting partition of $\mathcal{B}(M)$ is the one given by the preimages of the map e.

Proof. Let us first see that $\alpha$ is injective. Let $a \in \operatorname{Cob}^{*}(M, N)$ and $b \in$ $\operatorname{Cob}^{*}\left(M, N^{\prime}\right)$ with $N, N^{\prime} \in \mathcal{M}_{n}^{0}$. If $\alpha(a)=\alpha(b)$, then $e_{\circ} \alpha(a)=e_{\circ} \alpha(b)$ and then $N=N^{\prime}$, whence $a=b$ since $\alpha_{M, N}$ is injective. To prove the surjectivity of $\alpha$, let $\left(W, j_{M}, j_{N}\right)$ be an invertible cobordism and let $N_{0}$ be the representative of $e\left(\left[W[)\right.\right.$ in $\mathcal{M}_{n}^{0}$. Thus there exists a diffeomorphism $h: N_{0} \rightarrow N$ and $\left[W\left[=\alpha_{M, N_{0}}\left(\left[W, j_{M}, j_{N} \circ h\right]\right)\right.\right.$.

Remarks 3.9. (1) Composition of cobordisms defines an operation

$$
\operatorname{Cob}^{*}(L, M) \times \mathcal{B}(M) \stackrel{\circ}{\longrightarrow} \mathcal{B}(L),
$$

making $\mathcal{B}$ a functor on the category of closed manifolds and (equivalence classes of) invertible cobordisms.

(2) There is a version $\mathcal{B}^{\prime}(M)$ of $\mathcal{B}(M)$ where we only use triples $(W, M, N)$. The obvious inclusion $\mathcal{B}^{\prime}(M) \rightarrow \mathcal{B}(M)$ is, in fact, a bijection, by the observation at the end of 2.2. This will often be usedwithout further mention, to simplify notation.

Note that, using Lemma 2.4 the map $\mathcal{B}(M) \rightarrow \mathcal{B}^{\prime}(M)$ can also be defined as $\left[W, j_{M}, j_{N}\right] \mapsto\left[C_{j_{M}} \circ\left(W, \operatorname{id}_{M^{\prime}}, j_{N}\right)\right]$, where $M^{\prime}=j_{M}(M)$.

3.10. $h$-cobordisms. A cobordism $\left(W, j_{M}, j_{N}\right)$ from $M$ to $N$ is called an $h$ cobordism if both of the maps $j_{M}: M \rightarrow W$ and $j_{N}: N \rightarrow W$ are homotopy equivalences. The composition of $j_{N}$ with a homotopy inverse of $j_{N}$ then produces a homotopy equivalence $h: N \rightarrow M$ whose homotopy class is well defined. Any choice of such an $h$ will be called a natural homotopy equivalence associated to $W$. The main relationship between h-cobordisms and invertible cobordism is given by the following proposition. 
Proposition 3.11. An invertible cobordism is an h-cobordism. The converse is true when $n \neq 3$.

The above statement is unknown for $n=3$.

Proof. It suffices to consider the case of an invertible triad $(W, M, N)$. Let $\left(W^{\prime}, N, M\right)$ be an inverse for $W$, and choose diffeomorphisms $W \circ W^{\prime} \stackrel{\widetilde{\rightarrow}}{\rightarrow} M \times I$ rel $M$ and $W^{\prime} \circ W \stackrel{\approx}{\rightarrow} N \times I$ rel $N$. The inclusions $M \subset W \subset W \circ W^{\prime}$ and $W \subset W \circ W^{\prime} \subset W \circ\left(W^{\prime} \circ W\right) \approx W$ show that $M$ and $W$ are homotopy retracts of each other. Analogously for $N$ and $W$.

That an h-cobordism is invertible when $n \geq 5$ will be established in the proof of Theorem 3.15. For $n=4$, this is a result of Stallings (see [59, Thm 4]), and for $n \leq 2$ it follows from (the proof of) Proposition 6.3

3.12. Whitehead torsion. We recall here some facts about Whitehead torsion and the s-cobordism theorem. For more details, see [50, 9].

The Whitehead group $\mathrm{Wh}(\pi)$ of a group $\pi$ is defined as

$$
\mathrm{Wh}(\pi)=G L_{\infty}(\mathbb{Z} \pi) / E_{\infty}(\mathbb{Z} \pi) \cup( \pm \pi),
$$

where $E_{\infty}(\mathbb{Z} \pi)$ is the subgroup of elementary matrices and $( \pm \pi)$ denote the subgroup of $(1 \times 1)$-invertible matrix $( \pm \gamma)$ with $\gamma \in \pi$. As $E_{\infty}(\mathbb{Z} \pi)$ is the commutator of $G L_{\infty}(\mathbb{Z} \pi)$, the group $\mathrm{Wh}(\pi)$ is abelian.

A pair $(X, Y)$ of finite connected CW-complexes is an $h$-pair if the inclusion $Y \hookrightarrow X$ is a homotopy equivalence. To such a pair is associated its Whitehead torsion $\tau(X, Y) \in \mathrm{Wh}\left(\pi_{1} Y\right)$. The Whitehead torsion $\tau(f) \in \mathrm{Wh}(K)$ of a homotopy equivalence $f: K \rightarrow L$ ( $K, L$ finite $\mathrm{CW}$-complexes) is defined by $\tau(f)=\tau\left(C_{f}, K\right)$, where $C_{f}$ is the mapping cylinder of $f$. If $\tau(f)=0$, we say that $f$ is a simple homotopy equivalence.

If $K \stackrel{f}{\longrightarrow} L \stackrel{g}{\longrightarrow} M$ are homotopy equivalences between finite CW-complexes, then

$$
\tau(g \circ f)=\tau(f)+\left(f_{*}\right)^{-1}(\tau(g))
$$

where $f_{*}: \mathrm{Wh}\left(\pi_{1} L\right) \rightarrow \mathrm{Wh}\left(\pi_{1} K\right)$ is the isomorphism induced by $f$. Also useful is the following partial product formula. Let $K, L$ and $Z$ be connected finite CWcomplexes and let $f: K \rightarrow L$ be a homotopy equivalence. Then, in $\operatorname{Wh}\left(\pi_{1}(K \times\right.$ $Z)$ ), one has

$$
\tau\left(f \times \operatorname{id}_{Z}\right)=\chi(Z) \cdot \tau(f),
$$

where $\chi(Z)$ is the Euler characteristic of $Z$ (see [9, (23.2)]).

Remark 3.13. This definition of the torsion of a homotopy equivalence is slightly non-standard, as it measures the torsion in the Whitehead group of the source of $f$, rather than the target, as in [9] and [50]. The two definitions are of course equivalent, but for our purposes, the current definition is more convenient, since now the torsion of a pair $(X, Y)$ is equal to the torsion of the inclusion map $X \subset Y$. 
An easy case for computing $\tau(X, Y)$ is when the h-pair $(X, Y)$ is in simplified form, i.e.

$$
X=Y \cup \bigcup_{i=1}^{p} e_{i}^{r} \cup \bigcup_{i=1^{p}} e_{i}^{r+1} \quad(r \geq 2)
$$

where $e_{i}^{j}$ denotes a $j$-cell. Let $(\tilde{X}, \tilde{Y})$ be the pair of universal covers. Then the chain complex of $C_{*}(\tilde{X}, \tilde{Y})$ is a complex of free $\mathbb{Z} \pi$-modules and the boundary operator $\delta: C_{r+1}(\tilde{X}, \tilde{Y}) \rightarrow C_{r}(\tilde{X}, \tilde{Y})$ is an isomorphism. Bases may be obtained for $C_{*}(\tilde{X}, \tilde{Y})$ by choosing orientations of $e_{i}^{j}$ and liftings $\tilde{e}_{i}^{j}$ in $\tilde{X}$. Then,for such bases, $\tau(X, Y)$ is represented in $G L_{p}(\mathbb{Z} \pi)$ by the matrix of $\delta^{\varepsilon}$ with $\varepsilon=(-1)^{(r-1)}$.

Let $M$ be a connected manifold. The Whitehead group $\mathrm{Wh}\left(\pi_{1} M\right)$ is then endowed with an involution

$$
\tau \mapsto \bar{\tau}
$$

induced by the anti-automorphism of $\mathbb{Z} \pi_{1} M$ satisfying $\bar{a}=\omega(a) a^{-1}$ for $a \in$ $\pi_{1} M$, where $\omega: \pi_{1} M \rightarrow\{ \pm 1\}$ is the orientation character of $M$. We denote by $\mathrm{Wh}(M)$ the abelian group $\mathrm{Wh}\left(\pi_{1} M\right)$ equipped with this involution.

Let $W$ be an invertible cobordism starting from the closed connected manifold $M$. Then $(W, M)$ admits a $\mathcal{C}^{1}$-triangulation which is unique up to PLhomeomorphism [69, Theorems 7 and 8]. This makes $(W, M)$ an h-pair whose Whitehead torsion $\tau(W, M) \in \mathrm{Wh}(M)$ is well defined. An invertible cobordism with vanishing torsion is called an $s$-cobordism.

To compute $\tau(W, M)$, one can use a simplified form analogous to 3.13).

Lemma 3.14. Let $(W, M, N)$ be an invertible cobordism with $\operatorname{dim} M=n \geq 4$. Then, for $2 \leq r \leq n-2$, there exists a decomposition

$$
W=W_{r} \circ W_{r+1}
$$

where $\left(W_{r}, M, M_{r}\right)$ has a handle decomposition starting form $M$ with only handles of index $r$ and $\left(W_{r+1}, M_{r}, N\right)$ has a handle decomposition starting form $M_{r}$ with only handles of index $r+1$.

Proof. When $n \geq 5$, this is [32, Lemma 1]. We have to see that the proof works for $n=4$. The principle is to eliminate handles of index $k$ by replacing them by handles of index $k+2$. There is an easy argument eliminating 0-handles, which also works when $n=4$. There is also a special argument to get rid of 1-handles, given in [32, pp. 35-36]. This argument also works when $n=4$ : it suffices to prove that two embeddings $f_{0}, f_{1}$ of $S^{1}$ into a 4-dimensional manifold $P$ which are related by a homotopy $f_{t}$ are ambient isotopic. Let $f: S^{1} \times I \rightarrow P \times I$ be the map $\left.f(x, t)=\left(f_{t}(x), t\right)\right)$. By general position, $f$ is homotopic relative $S^{1} \times \partial I$ to an embedding. Therefore, $f_{0}$ and $f_{1}$ are concordant and, as we are in codimension 3, they are ambient-isotopic [27].

The number of handles for $W_{r+1}$ and $W_{r}$ is the same (say, $p$ ) since $M \hookrightarrow W$ is a homotopy equivalence. As a consequence (see [54, p. 83]), $(W, M)$ retracts by deformation relative $M$ onto a CW-pair $(X, M)$ as in (3.13) from which we can compute $\tau(W, M)=\tau(X, M)$. 
Torsions of invertible cobordisms satisfy some specific formulae. First, let $(W, M, N)$ and $\left(W^{\prime}, N, N^{\prime}\right)$ be invertible cobordisms. Then, in $\mathrm{Wh}(M)$, one has

$$
\tau\left(W \circ W^{\prime}, M\right)=\tau(W, M)+h_{*}\left(\tau\left(W^{\prime}, N\right)\right),
$$

where $h_{*}: N \rightarrow M$ is a natural homotopy equivalence associated to $W$. This follows from [9, (20.2) and (20.3)]. One also has the duality formula (see [50, pp. 394-398]):

$$
h_{*}(\tau(W, N))=(-1)^{n} \overline{\tau(W, M)} .
$$

More generally, if $\left(W, j_{M}\right)$ represents an element in $\mathcal{B}(M)$, we define

$$
\mathcal{T}\left(W, j_{M}\right)=\tau\left(j_{M}\right)=\left(j_{M_{*}}\right)^{-1} \tau\left(W, j_{M}(M)\right) .
$$

The duality formula now becomes

$$
\left(j_{N}\right)_{*}^{-1}\left(j_{M}\right)_{*}\left(\tau\left(W, j_{N}\right)\right)=(-1)^{n} \overline{\tau\left(W, j_{M}\right)} .
$$

Thanks to the uniqueness of $\mathcal{C}^{1}$-triangulations, this gives a well defined map

$$
\mathcal{T}: \mathcal{B}(M) \rightarrow \mathrm{Wh}(M) .
$$

Theorem 3.15. Let $M$ be a smooth closed connected manifold of dimension $\geq 5$. Then,

(i) the map $\mathcal{T}: \mathcal{B}(M) \rightarrow \mathrm{Wh}(M)$ is a bijection;

(ii) $\mathcal{T}\left(W, j_{M}\right)=0$ if and only if $\left(W, j_{M}(M)\right) \approx_{\text {diff }}(M \times I, M \times 0)(\operatorname{rel} M)$.

For the situation when $n=3,4$, see Lemma 5.8, the end of Section 5 and Section 6 .

Proof. We decompose the proof into three steps.

(1) Part (ii). This is the content of the s-cobordism theorem, which is valid for $n \geq 5$. This theorem was first independently proved by Barden, Mazur and Stallings in the early 60's. For a proof and references, see [32].

(2) Surjectivity of $\mathcal{T}$. Let $\pi=\pi_{1} M$. For any element $\sigma \in \mathrm{Wh}(\pi)$ there is an h-cobordism $(W, M, N)$ with $\tau(W, M)=\sigma$ (see [50, Theorem 11.1]). Thus, to prove the surjectivity of $\mathcal{T}$, it is enough to prove that $W$ is invertible. Let $f: N \rightarrow M$ be the composition of the inclusion $N \hookrightarrow W$ with a retraction from $W$ to $M$. Let $\left(W_{R}, N, M_{R}\right)$ be an h-cobordism such that $f_{*}\left(\tau\left(W_{R}, N\right)\right)=-\sigma$. By (3.15), one has

$$
\tau\left(W \circ W_{R}, M\right)=\tau(W, M)+f_{*}\left(\tau\left(W_{R}, N\right)\right)=0 .
$$

By Part (ii) already established, there exists a diffeomorphism (relative $M$ ) $H: W \circ W_{R} \rightarrow M \times I$. Let $h: M_{R} \rightarrow M \times 1$ be the restriction of $H$ to $M_{R}$. Using the diffeomorphism $H$ and Lemma 2.4 one gets

$$
\left[W \circ W_{R}, j_{M} \amalg j_{M_{R}}\right]=\left[M \times I, j_{M}^{0} \amalg h\right]=\left[C_{h}\right] .
$$


Therefore, $[W] \circ\left[\hat{W}_{R}\right]=\mathbf{1}_{M}$, where $\left[\hat{W}_{R}\right]=\left[W_{R}\right] \circ\left[C_{h^{-1}}\right]$.

Similarly, let $\left(W_{L}, M_{L}, M\right)$ be an h-cobordism with $\tau\left(W_{L}, M\right)=(-1)^{n+1} \bar{\sigma}$. $\mathrm{By}(3.16)$ and 3.15 , one has

$f_{*}\left(\tau\left(W_{L} \circ W, N\right)\right)=f_{*}\left(\tau(W, N)+f_{*}^{-1}\left(\tau\left(W_{L}, M\right)\right)\right)=(-1)^{n} \bar{\sigma}+(-1)^{n+1} \bar{\sigma}=0$.

As above, this permits us to construct a cobordism $\hat{W}_{L}$ from $N$ to $M$, such that $\hat{W}_{L}$ is a left inverse for $W:\left[\hat{W}_{L}\right]_{\circ}[W]=\mathbf{1}_{N}$. Having a left and right inverse, $[W]$ is invertible and $\left[W_{L}\right]=\left[W_{R}\right]$.

Remark: we have proven in (2) that any h-cobordism is invertible if $\operatorname{dim} M \geq 5$.

(3) Injectivity of $\mathcal{T}$. Let $(W, M, N)$ and $\left(W^{\prime}, M, N^{\prime}\right)$ be two invertible cobordisms starting from $M$, with $\tau(W, M)=\tau\left(W^{\prime}, M\right)=\alpha$. Let $(V, P, M)$ be an h-cobordism such that $\tau(V, M)=(-1)^{n+1} \bar{\alpha}$. As in (3.18), we check that $\tau(V \circ W, P)=\tau\left(V \circ W^{\prime}, P\right)=0$. By (1) above, there are diffeomorphisms (relative $P$ ) $H: V \circ W \rightarrow P \times I$ and $H^{\prime}: V \circ W^{\prime} \rightarrow P \times I$, with restrictions $h: N \rightarrow P \times 1 \approx P$ and $h^{\prime}: N^{\prime} \rightarrow P \times 1 \approx P$. Then

$$
[V] \circ\left[W \left[=\left[P \times I\left[=[V] \circ\left[W^{\prime}[.\right.\right.\right.\right.\right.
$$

As $[V]$ is invertible, one gets the equality $\left[W\left[=\left[W^{\prime}[\right.\right.\right.$ in $\mathcal{B}(M)$.

Remark 3.16. The results of this section may be used to give an alternative proof that two closed manifolds $M$ and $N$ of dimension $\geq 4$ which are $h$-cobordant are $\mathbb{R}$-diffeomorphic (Proposition 3.3). Indeed, let $(W, N, M)$ be an h-cobordism Then, $W \times S^{1}$ is an s-cobordism by $(3.12$ and thus, using Theorem 3.15, there exists a diffeomorphism $F: N \times S^{1} \times I \rightarrow W \times S^{1}$ inducing a diffeomorphism $F^{1}: N \times S^{1} \times 1 \rightarrow M \times S^{1}$. By Proposition 3.3, one deduces that $M \approx_{\mathbb{R} \text { diff }} N$. Indeed, Condition (c) of Proposition 3.3 may be checked for $\beta=F_{1}$, using that $F$ may be chosen relative $N \times S^{1} \times 0$.

\subsection{Remarks on the relative case. Concordance.}

With minor modifications most of the results in this section go through also in the relative case, i. e. when $M$ and $N$ have nonempty boundaries. In particular, we can define invertible cobordisms and relative invertible cobordisms the same way in this generality. Moreover, the crucial results used in this section, the s-cobordism theorem and classification of h-cobordisms by Whitehead torsion still hold. Although they are usually only formulated in the closed case, the proofs don't really use this, but work exactly the same way in general, since all the constructions can be done 'away from the boundary'. This means that Theorem 3.15 could just as well have been formulated for manifolds with boundary, to the expense of a little more notation.

Here we will not need a full discussion of this, but in Section 7 we come back to a special case, when we wish to compare invertible cobordisms between the same manifold, using the relation of concordance.

Fix two invertibly cobordant closed, compact manifolds $M$ and $N$, and let $\left(W, j_{M}, j_{N}\right)$ and $\left(W^{\prime}, j_{M}^{\prime}, j_{N}^{\prime}\right)$ be two invertible cobordisms between them. We say that these cobordisms are concordant if there is an invertible cobordism 
$\left(X, J_{W}, J_{W^{\prime}}\right)$ between them, with the following extra compatibility condition between $J_{*}$ 's and $j_{*}$ 's: There are embeddings $H_{M}: M \times I \rightarrow \partial X$ and $H_{N}$ : $N \times I \rightarrow \partial X$ filling in $\partial X-\left(J_{W} W \cup J_{W^{\prime}} W^{\prime}\right)$ and such that $J_{W} j_{M}=H_{M} j_{M}^{0}$, $J_{W^{\prime}} j_{M}^{\prime}=H_{M} j_{M}^{1}, J_{W} j_{N}=H_{M} j_{N}^{0}$ and $J_{W^{\prime}} j_{N}^{\prime}=H_{N} j_{N}^{1}$.

Observe that concordance defines an equivalence relation on $\operatorname{Cob}^{*}(M, N)$. We denote the set of equivalence classes by $\overline{C_{o b}}(M, N)$. Via the composed map $\operatorname{Cob}^{*}(M, N) \rightarrow \mathcal{B}(M) \rightarrow \mathrm{Wh}(M)$ this relation corresponds to a relation on $\mathrm{Wh}(M)$, which will be important in Section 7 .

Lemma 3.18. Let $M$ and $N$ be a compact closed manifolds of dimension $n$, let $\left(W, j_{M}, j_{N}\right)$ and $\left(W^{\prime}, j_{M}^{\prime}, j_{N}^{\prime}\right)$ be two invertible cobordisms, and assume $\left(X, J_{W}, J_{W^{\prime}}\right)$ is a concordance between them. The Whitehead torsions are then related by the formula

$$
\tau\left(W^{\prime}, j_{M}^{\prime}\right)-\tau\left(W, j_{M}\right)=j_{M_{*}^{-1}}\left(\tau\left(X, J_{W}\right)+(-1)^{n} \overline{\tau\left(X, J_{W}\right)}\right) .
$$

Proof. The two maps $j_{W} j_{M}$ and $j_{W^{\prime}} j_{M}^{\prime}$ are homotopic homotopy equivalences. Hence they have the same torsion, and we get the identity

$$
\tau\left(j_{M}\right)+j_{M_{*}^{*}}^{-1}\left(\tau\left(j_{W}\right)\right)=\tau\left(j_{M}^{\prime}\right)+j_{M *}^{\prime-1}\left(\tau\left(j_{W^{\prime}}\right)\right) .
$$

The result now follows from the duality formula $(\sqrt{3.17})$.

\section{The case $n \geq 5$}

The following theorem is a direct consequence of Proposition 3.3 and Theorem 3.15 .

Theorem 4.1. Let $M$ and $N$ be smooth closed connected manifolds of dimension $n \geq 5$ such that $N \approx_{\mathbb{R} \text {-diff }} M$. Suppose that $\mathrm{Wh}(M)=0$. Then $N \approx_{\text {diff }} M$.

As $\operatorname{Wh}(\{1\})=0$, Theorem 4.1 implies Theorem A in the case $n \geq 5$. As a first generalization, let us consider the following conjecture.

Conjecture 4.2. Let $M$ and $N$ be smooth connected closed manifolds of dimension $\geq 5$ such that $N \approx_{\mathbb{R} \text {-diff }} M$. Suppose that $\pi_{1} M$ is torsion-free. Then $N \approx_{\text {diff }} M$.

Using Theorem 4.1. Conjecture 4.2 would follow from the well known conjecture that $\mathrm{Wh}(\pi)=0$ if $\pi$ is a torsion-free finitely presented group. This is part of the Farrell-Jones conjecture in K-theory and it has been proven by several authors for various classes of finitely presented torsion-free groups, such as free abelian groups, free groups, virtually solvable groups, word-hyperbolic

groups, CAT(0)-groups, etc. For references, see [46, 2] (see also the proof of Theorem 5.1).

To generalize Theorem 4.1 we need to introdue the concept of inertial invertible cobordisms: a cobordism $\left(W, j_{M}, j_{N}\right)$ is inertial if $N \approx_{\text {diff }} M$. 
Let $\mathcal{I B}(M)$ be the subset of elements in $\mathcal{B}(M)$ represented by inertial cobordisms and let $I(M)=\mathcal{T}(\mathcal{I B}(M)) \subset \mathrm{Wh}(M)$. Note that $I(M)$ is not a subgroup of $\mathrm{Wh}(M)$ in general [19, Remark 6.2].

Theorem 3.15 together with Proposition 3.3 implies the following result, which is the strongest possible generalization of Theorem 4.1 .

Theorem 4.3. For $M$ a smooth connected closed manifold of dimension $\geq 5$, the following assertions are equivalent.

(i) Any manifold $\mathbb{R}$-diffeomorphic to $M$ is diffeomorphic to $M$.

(ii) $I(M)=\mathrm{Wh}(M)$.

The set $I(M)$ is contained in the set $I_{\mathrm{TOP}}(M)$ of those $\sigma \in \mathrm{Wh}(M)$ such that if $(W, M, N)$ is an invertible cobordism with $\tau(W, M)=\sigma$, then $N \approx_{\text {top }} M$. In all cases where these sets are computed, they are equal, but it is not known whether $I(M)=I_{\mathrm{TOP}}(M)$ in general for a smooth manifold $M$ of dimension $\geq 5$, contrary to the claim in 29 . However, there is a smaller set, $S I(M)$, of strongly inertial invertible cobordisms, which indeed is the same in the two categories. This is the set of invertible cobordisms $\left(W, j_{M}, j_{N}\right)$ such that $j_{M}^{-1} \circ j_{N}$ is homotopic to a diffeomorphism (homeomorphism). See [30].

The general question is intriguing, not the least because of the following reformulation:

Question 4.4. Given two smooth manifolds $M$ and $N$ of dimension $\neq 4$ such that $M \approx_{\mathbb{R} \text {-diff }} N$ and $M \approx_{\text {top }} N$. Is $M \approx_{\text {diff }} N$ ?

The answer of the above question is "infinitely no" in dimension 4, even if $M$ and $N$ are simply connected (see Section 5). It is "yes" in dimension 3 for orientable manifolds (see Theorem C).

Examples 4.5. We start with examples where $I(M) \neq \mathrm{Wh}(M)$.

(1) $I_{\mathrm{TOP}}(M) \neq \mathrm{Wh}(M)$ for $M=L(7,1) \times S^{4}$ or $M=L(7,2) \times S^{4}$. Indeed, in 1961, J. Milnor [48] showed that these two manifolds are invertibly cobordant but have not the same simple homotopy type (they are then not homeomorphic by Chapman's theorem [9, Appendix]). Historically, this was the first example of this kind and Milnor used it to produce the first counterexample to the Hauptvermutung for finite simplicial complexes [48].

(2) $I_{\mathrm{TOP}}(M)=0$ if $M$ is a lens space of dimension $\geq 5$ [50, Corollary 12.13]. This result was extended in [39] to generalized spherical spaceforms (see 8.6).

(3) For $k \geq 3$, one has $I_{\mathrm{TOP}}\left(L(p, q) \times S^{2 k}\right)=0$ if $p \equiv 3(\bmod 4)$. Also, $I\left(L(5,1) \times S^{2 k}\right)=0$ but there exists a manifold $N h$-cobordant to $L(5,1) \times S^{2 k}$ such that $I(N) \neq 0$ (see [19, $\S 6]$ ).

(4) Let $W$ be an invertible cobordism and consider its dual $\bar{W}$ (see 2.2). Then, $W \circ \bar{W}$ is an inertial invertible cobordism. By (3.15) and (3.16), one has $\tau(W \cup \bar{W}, M)=\tau(W, M)+(-1)^{n} \overline{\tau(W, M)}$. Therefore $\mathcal{N}(M)=\{\tau+$ $\left.(-1)^{n} \bar{\tau} \mid \tau \in \mathrm{Wh}(M)\right\} \subset I(M)$. The subgroup $\mathcal{N}(M)$ plays an important role in Section 7 . 
(5) Let $\pi$ be a finite group such that $\mathrm{Wh}(\pi)$ is infinite. (For $\pi$ abelian, this is the case unless $\pi$ has exponent $2,3,4$ or 6 : see [3]). Then, in every odd dimension $\geq 5$, there are manifolds $M$ with fundamental group $\pi$ such that $I_{\mathrm{TOP}}(N)$ is finite for any manifold $N$ invertibly cobordant to $M$ (see 29, Theorem 1.2 and its proof]). Then there are infinitely many distinct homeomorphism classes of manifolds $\mathbb{R}$-diffeomorphic to $M$.

In view of Theorem 4.3 , the case $I(M)=\mathrm{Wh}(M)$ is particularly interesting. The proof of the following proposition uses a standard technique to produce $h$-cobordisms, going back to [48, $\S 2]$ and generalized independently in [43] and [18.

Proposition 4.6. Let $K$ be a finite 2-dimensional polyhedron with $\pi_{1} K$ finite abelian and let $n \geq 5$. Let $E$ be a regular neighborhood of an embedding of $K$ in $\mathbb{R}^{n+1}$ and let $M=\partial E$. Then $I(M)=\mathrm{Wh}(M)$.

Proof. Let $i: K \rightarrow E$ be the natural inclusion and let $f: K \rightarrow K$ be a homotopy equivalence with homotopy inverse $\varphi$. Then, $i \circ f$ is homotopic to an embedding $j_{f}: K \rightarrow E$. Let $V_{f}$ be a regular neighborhood of $j_{f}(K)$ in $E$ and let $W_{f}=$ $E-\operatorname{int} V_{f}$. Doing the same construction in $V_{f}$ with $j_{f} \circ \varphi$, and another time using again $f$, shows that $\left(W_{f}, \partial V_{f}, M\right)$ is an invertible cobordism. The torsion of $W_{f}$ is related to $\tau(f)$, via natural identifications of fundamental groups (see [18, proof of Proposition 1.1] or [43, Proposition 3]). As $j_{f}$ is isotopic to $i$ in $\mathbb{R}^{n+1}$, one has $E \approx_{\text {diff }} V_{f}$, thus $W_{f}$ is inertial. By [42, Theorem 1], every element of $\mathrm{Wh}\left(\pi_{1} K\right)$ is realizable as the torsion of a self homotopy equivalence of $K$. This proves that $I(M)=\mathrm{Wh}(M)$.

In the even case, this result has a vast generalization, as a consequence of the following Proposition.

Proposition 4.7. Let $M$ be a smooth connected closed manifold of dimension $n \geq 5$. Let $\sigma \in \mathrm{Wh}(M)$ such that $\sigma=(-1)^{n} \bar{\sigma}$. Then $\sigma \in I(M)$.

Proof. Let $i: K \rightarrow M$ be an embedding of a finite connected 2-dimensional complex $K$ into $M$ such that $\pi_{1} i: \pi_{1}(K) \rightarrow \pi_{1}(M)$ is an isomorphism, which we use to measure Whitehead torsions in $\pi_{1}(K)$. Let $A$ be a regular neighborhood of $i(K)$ and let $B=M-\operatorname{int} A$.

Let $\left(V, A, A^{\prime}\right)$ be an invertible cobordism relative boundary with $\tau(V, A)=\sigma$. Then, $W=V \cup(B \times I)$ is an invertible cobordism from $M$ to $M^{\prime}=A^{\prime} \cup(B \times 1)$ with $\tau(W, M)=\sigma$.

Since $\operatorname{dim} M \geq 5$ and codim $K \geq 3$, we have $\operatorname{dim} \partial A \geq 4$ and $\pi_{1} \partial A=\pi_{1} A$. Then, by Theorem 3.11 and Lemma 5.6, there also exists an invertible cobordism $T \in \mathcal{B}(\partial A)$ with Whitehead torsion $\sigma$. The condition $\sigma=(-1)^{n} \bar{\sigma}$ now means that $T^{-1}=\bar{T}$, and $A \circ T \circ \bar{T} \approx_{\text {diff }} A$, rel $\partial$.

Let $C=A \circ T$. Then we may also consider $V$ as an h-cobordism from $C$ to $A^{\prime} \circ T$, and computing the torsion of the inclusion $K \subset V$ two ways, we see that $\tau(V, C)=0$. By the s-cobordism theorem we conclude that $C \approx_{\text {diff }} A^{\prime} \circ T$ rel $\partial$, and hence $A^{\prime} \approx_{\text {diff }} A$ rel $\partial$, since $T$ is invertible. Extending this diffeomorphism by the identity on $B$, we see that $M^{\prime} \approx_{\text {diff }} M$. 
(Remark: When $\sigma \neq(-1)^{n} \bar{\sigma}$, it is still possible that $M^{\prime} \approx_{\text {diff }} M$, as seen above; simply, the diffeomorphism from $M^{\prime}$ to $M$ is not relative $B$.)

When $M$ is orientable with $\pi_{1} M$ finite abelian, then $\bar{\sigma}=\sigma$ for all $\sigma \in$ $\mathrm{Wh}(M)$ [1, whence the following corollary of Proposition 4.7 .

Corollary 4.8. Let $M$ be a connected orientable closed manifold of even dimension $\geq 6$ such that $\pi_{1} M$ finite abelian. Then $I(M)=\mathrm{Wh}(M)$.

In the case when $\pi_{1}(M)$ is finite cyclic, this was first proved in [43, Cor. 1]

We also mention another corollary of Proposition 4.7, which essentially amounts to a curious reformulation. Let $(W, M, N)$ be an invertible cobordism with Whitehead torsion $\sigma=\tau(W, M)$, and let $h: N \rightarrow M$ be a natural homotopy equivalence associated to $W$. It follows easily from the composition and duality formulae (3.11) and (3.16) that $\tau(h)=-\sigma+(-1)^{n} \bar{\sigma}$. Hence we see that $h$ is a simple homotopy equivalence if and only if $\sigma=(-1)^{n} \bar{\sigma}$.

Corollary 4.9. If the natural homotopy equivalence defined by the invertible cobordism $(W, M, N)$ is simple, then $(W, M, N)$ is inertial.

But note that $h$ may not itself be homotopic to a homeomorphism! A counterexample is given in [29, Example 6.4].

Finally, we describe how to get inertial invertible cobordisms by "stabilization" (up to connected sums with $S^{r} \times S^{n-r}$ ). First, a few words about connected sums. Since we do not worry about orientations, the diffeomorphism type $M_{1} \sharp M_{2}$ may depend on the choice of embeddings $\beta_{i}$ : $D^{n} \rightarrow M_{i}$ (see e.g. [20, $§ 4.2 .3]$ ). This will not bother us because our manifold $M_{2}$ (like $S^{r} \times S^{n-r}$ ) admits an orientation reversing diffeomorphism. The same holds true for cobordism connected sum $W_{1} \sharp W_{2}$, obtained using embeddings $\beta_{i}:\left(D^{n} \times I, D^{n} \times 0, D^{n} \times 1\right) \rightarrow\left(W_{i}, M_{i}, N_{i}\right)$.

Proposition 4.10. ([17, compare 8.5) Let $M$ be a smooth connected closed manifold of dimension $n \geq 5$. Let $(W, M, N)$ ) be an invertible cobordism such that $\tau(W, M)$ is represented by a matrix in $G L_{p}\left(\mathbb{Z} \pi_{1} M\right)$. Then, for $2 \leq r \leq$ $n+2$,

$$
M \sharp p\left(S^{r} \times S^{n-r}\right) \approx_{\text {diff }} N \sharp p\left(S^{r} \times S^{n-r}\right) .
$$

Consequently, the cobordism $W \sharp p\left(S^{r} \times S^{n-r} \times I\right)$ is an inertial invertible cobordism.

Proof. One uses a simplified handle decomposition $W=W_{r} \circ W_{r+1}$ like in Lemma 3.14, together with the remark of [17] that the $r$-handles of $\left(W_{r}, M, M_{r}\right)$ are attached trivially, meaning that the attaching embedding factors through the standard embedding of $S^{r-1} \times D^{n+1-r}$ into $\mathbb{R}^{n}$. This implies that $M_{r} \approx_{\text {diff }}$ $M \sharp p\left(S^{r} \times S^{n-r}\right)$. The same holds true for the $(n-r)$-handles of $\left(\bar{W}_{r+1}, N, M_{r}\right)$, thus $M \sharp p\left(S^{r} \times S^{n-r}\right)$. For details, see [17].

Combined with Proposition 3.3, this gives an interesting relation between two kinds of stabilization: 
Corollary 4.11. Let $M$ and $N$ be closed smooth manifolds of dimensions $\geq 5$ which are $\mathbb{R}$-diffeomorphic. Then there exists an integer $p$ such that $M \sharp p\left(S^{r} \times\right.$ $\left.S^{n-r}\right) \approx_{\text {diff }} N \sharp p\left(S^{r} \times S^{n-r}\right)$ for any $r$ such that $2 \leq r \leq n-2$. If $\pi_{1}(M)$ is finite, $p$ may be chosen $\leq 2$.

Proof. The last statement follows since $G L_{2}(\mathbb{Z} \pi) \rightarrow \mathrm{Wh}(\pi)$ is surjective if $\pi$ is a finite group 63]. Note that $p$ can not always be chosen to be 1 (see [29, Theorem 1.1]).

An intriguing question is if there is some kind of converse to this result. A very special case is given by Lemma 4.1 in 29 .

By Theorem 3.15, an invertible cobordism $X$ starting from $Y=M \sharp p\left(S^{r} \times\right.$ $\left.S^{n-r}\right)$ is of the form $W \sharp p\left(S^{r} \times S^{n-r} \times I\right)$ where $W$ is an invertible cobordism starting from $M$ with $\tau(X, Y)=\tau(W, M)$. Using Proposition 4.10, this proves the following

Corollary 4.12. Let $M$ be a smooth connected closed manifold of dimension $n \geq 5$. Suppose that $G L_{p}\left(\mathbb{Z} \pi_{1} M\right) \rightarrow \mathrm{Wh}\left(\pi_{1} M\right)$ is surjective. Then, for any $2 \leq r \leq n+2$, one has $I\left(M \sharp p\left(S^{r} \times S^{n-r}\right)\right)=\mathrm{Wh}(M)$.

\section{The case $n=4$}

A group $\pi$ is called poly-(finite or cyclic) if it admits an ascending sequence of subgroups, each normal in the next, with successive quotients either finite or cyclic (this is equivalent to $\pi$ being virtually polycyclic: see [67, Theorem 2.6]). We first prove the following theorem which implies Part (ii) of Theorem A.

Theorem 5.1. Let $M$ and $N$ be smooth connected closed manifolds of dimension 4 such that $N \approx_{\mathbb{R} \text {-diff }} M$. Suppose that $\pi_{1} M$ is poly-(finite or cyclic) and that $\mathrm{Wh}(M)=0$. Then $N \approx_{\text {top }} M$.

Proof. By Proposition 3.3, there is an invertible cobordism $W$ from $M$ to $N$. Then $W$ is an h-cobordism by Proposition 3.11 and, as $\mathrm{Wh}(M)=0$, it is an scobordism. The topological s-cobordism theorem in dimension 4 holds for closed manifold with poly-(finite or cyclic) fundamental group [14, Theorem 7.1A and the Embedding theorem p. 5]. Therefore, $W \approx_{\text {top }} M \times I(\operatorname{rel} M)$ and then $N \approx_{\text {top }} M$.

Example 5.2. By [12], $\mathrm{Wh}(M)=0$ when $\pi_{1} M$ is poly-(finite or cyclic) and torsion-free. By Theorem 5.1. $N \approx_{\mathbb{R} \text {-diff }} M$ implies $N \approx_{\text {top }} M$ in this case.

Remark 5.3. Poly-(finite or cyclic) groups are the only known examples of finitely presented groups which are called "good" by Freedman and Quinn, i.e. for which their techniques work [14, p. 99]. Freedman and Teichner [15] showed that groups of subexponential growth are good, but the only known such groups which are finitely presented are poly-(finite or cyclic). Note that Theorem 5.1 may be true even if $\pi_{1}(M)$ is not good in the above sense. 
We now prepare the proof of Theorem B of the introduction. Recall that, to a homeomorphism $f: M \rightarrow N$ between manifolds is associated its Casson-Sullivan invariant $\operatorname{cs}(f) \in H^{3}\left(M ; \mathbb{Z}_{2}\right)$, which, for $\operatorname{dim} M \geq 4$, vanishes if and only if $h$ is isotopic to a PL-homeomorphism (thus, to a diffeomorphism if $\operatorname{dim} M=4$ : see [55, Definition 3.4.5]).

Proposition 5.4. Let $M, N$ be two closed smooth connected 4-manifolds. Suppose that there exists a homeomorphism $f: M \rightarrow N$ with vanishing CassonSullivan invariant. Then, $M$ and $N$ are smoothly s-cobordant. The converse is true when $\pi_{1}(M)$ is poly-(finite or cyclic).

Proof. The mapping cylinder $C_{f}$ produces a topological s-cobordism $W$ between $M$ and $N$. As $\operatorname{dim} W=5$, the only obstruction to extend the smooth structure on $\partial W$ to a smooth structure on $W$ is the Kirby-Siebenmann class $\operatorname{ks}(W, \partial W) \in$ $H^{4}\left(W, \partial W ; \mathbb{Z}_{2}\right)$ (see [14, Theorem 8.3.B]). The image of $\operatorname{ks}(W, \partial W)$ under the isomorphism

$$
H^{4}\left(W, \partial W ; \mathbb{Z}_{2}\right) \approx H_{1}\left(W, \mathbb{Z}_{2}\right) \approx H_{1}\left(M ; \mathbb{Z}_{2}\right) \approx H^{3}\left(M ; \mathbb{Z}_{2}\right)
$$

coincides with $\operatorname{cs}(f)$ [55, Remark 3.4.6].

Conversely, let $(W, M, N)$ be a smooth s-cobordism. If $\pi_{1}(M)$ is poly-(finite or cyclic), the topological s-cobordism holds true (see the proof of Theorem 5.1). Therefore, $W \approx_{\text {top }} M \times I(\operatorname{rel} M)$ and the topological version of Example 3.7 makes $W$ homeomorphic rel $M$ to the mapping cylinder $C_{f}$ of a homeomorphism $f: M \rightarrow N$. Using (5.19), one has $\operatorname{cs}(f)=\operatorname{ks}(W, \partial W)=0$.

As $H^{3}\left(M ; \mathbb{Z}_{2}\right) \approx H_{1}\left(M ; \mathbb{Z}_{2}\right)$, one has the following corollary of Proposition 5.4 it was proven by C.T.C. Wall 65 when $M$ is simply connected, by a different method.

Corollary 5.5. Let $M$ and $N$ be smooth closed manifolds of dimension 4 which are homeomorphic. Suppose that $H_{1}\left(M, \mathbb{Z}_{2}\right)=0$. Then, $M$ and $N$ are smoothly s-cobordant.

We are ready to prove Theorem B of the introduction.

Proof of Theorem B. Let $M$ and $N$ be smooth closed manifolds of dimension 4 which are homeomorphic. By Corollary 5.5, there is a smooth h-cobordism $W$ between $M$ and $N$. Such a cobordism is invertible (see [59, Thm 4]; if $M$ is simply connected, then $W^{-1}=\bar{W}\left[54\right.$, Lemma 7.8]). Thus $N \approx_{\mathbb{R} \text {-diff }} M$ by Proposition 3.3 .

We now discuss a partial analogue to Proposition 4.10 which was first proven by C.T.C Wall in the simply connected case [65, Theorem 3] (compare 8.5).

Proposition 5.6. Let $M$ and $N$ be smooth closed connected manifolds of dimension 4 which are $\mathbb{R}$-diffeomorphic. Then, there exists $p \in \mathbb{N}$ such that

$$
M \sharp p\left(S^{2} \times S^{2}\right) \approx_{\text {diff }} N \sharp p\left(S^{2} \times S^{2}\right) .
$$


Proof. A simplified handle decomposition $W=(M \times I) \circ W_{2} \circ W_{3}$ like in Lemma 3.14 is available but we do not know that the 2-handles of $\left(W_{2}, M \times 1, M_{2}\right)$ are attached trivially (see [66, Theorem 3 and its proof]). However, since $\pi_{1}(M) \approx$ $\pi_{1}(W)$, the attaching map $\alpha: S^{1} \times D^{3} \rightarrow M \times 1$ of a 2 -handle of $W_{2}$ is homotopically trivial. As in the proof of Lemma 3.14 , this implies, using an ambient isotopy of $M \times 1$, that one may assume that $\alpha\left(S^{1} \times D^{3}\right)$ is contained in a disk. Also, $\alpha$ : $S^{1}=S^{1} \times 0 \rightarrow M \times 1$ extends to an embedding $\alpha_{-}: D^{2} \rightarrow M \times I$ and thus to an embedding $\bar{\alpha}: S^{2} \rightarrow W$. Since $\pi_{2}(M \times I) \rightarrow \pi_{2}(W)$ is an isomorphism, one can choose $\alpha_{-}$so that $\bar{\alpha}$ is homotopically trivial.

That $\alpha$ is attached trivially is thus equivalent to the triviality of the normal bundle $\nu$ to $\bar{\alpha}$. As a vector bundle over $S^{2}$, the Whitney sum $T S^{2} \oplus \nu$ is isomorphic to $\bar{\alpha}^{*} T W$. The latter is trivial since $\bar{\alpha}$ homotopically trivial. As $T S^{2}$ is stably trivial, so is $\nu$, which implies that $\nu$ is trivial since $\operatorname{rank} \nu>\operatorname{dim} S^{2}$.

Unlike in Proposition 4.10, the torsion of an invertible cobordism between $M$ and $N$ only furnishes a lower bound for the integer $p$ of Proposition 5.6, as seen by the case where $M$ and $N$ are simply connected. An interesting question would be to find the minimal integer $p$ necessary to construct a given invertible cobordism. Some results in the simply connected case may be found in [44].

We finish this section by considering the following problem which is important in view of Section 7

Problem 5.7. Describe the set $\mathcal{B}(M)$ for $M$ a smooth closed connected manifold of dimension 4.

Only partial information is currently known about this problem. For instance, the map $\mathcal{T}: \mathcal{B}(M) \rightarrow \mathrm{Wh}(M)$ of Theorem 3.15, associating to an invertible cobordism $(W, M, N)$ its Whitehead torsion $\tau(W, M)$ is defined, and one has the following

Lemma 5.8. Let $M$ be a smooth closed connected manifolds of dimension 4. Then, the map $\mathcal{T}: \mathcal{B}(M) \rightarrow \mathrm{Wh}(M)$ is surjective.

Proof. It is said in [14, p. 102] that $\mathcal{T}$ is surjective, based on "the standard construction of h-cobordisms" with reference to [54, p. 90]. But, when $n=4$, this standard construction for $\sigma \in \mathrm{Wh}(M)$ only provides a cobordism $(W, M, N)$ such that the inclusion $M \hookrightarrow W$ is a homotopy equivalence with torsion $\sigma$. By Poincaré duality, one has $\left.0=H^{*}(W, M ; \mathbb{Z} \pi) \approx H_{*} W, N ; \mathbb{Z} \pi\right)$, where $\pi=$ $\pi_{1}(W) \approx \pi_{1}(M)$. This proves that $W$ is a semi-h-cobordism from $N$, that is to say that the inclusion $N \hookrightarrow W$ is homotopy equivalent to a Quillen plusconstruction (see [21]); thus $i_{*}: \pi_{1}(N) \rightarrow \pi$ is onto with perfect kernel $K$.

By [14, Theorem 11.1A], there exists a semi-s-cobordism $\left(W^{\prime}, N, N^{\prime}\right)$ with $\pi_{1}(M) \rightarrow \pi_{1}\left(W^{\prime}\right)$ onto with kernel $K$. Formula (3.16) may be used here, and thus $X=W \circ W^{\prime}$ is an h-cobordism with $\tau(X, M)=\sigma$. As an h-cobordism between closed 4-manifolds, $X$ is invertible [59, Thm 4].

Some information is available on $\mathcal{B}(M)$ when $M$ is simply connected. By Corollary 5.5. the map $e$ of (3.8) may be replaced by a surjective map $e: \mathcal{B}(M) \rightarrow$ 
$\mathcal{M}(M)$, where $\mathcal{M}(M)$ is the set of diffeomorphism classes of manifolds homeomorphic to $M$. This set may be infinite [13], and so does $\mathcal{B}(M)$. Let $\mathcal{M}^{0}(M)$ be a set of representatives of $\mathcal{M}(M)$. For $M$ oriented, one can precompose the bijection of Lemma 3.8 by the surjective map

$$
\bigsqcup_{N \in \mathcal{M}_{\mathrm{or}}^{0}(M)} \operatorname{Cob}^{*, \mathrm{or}}(M, N) / \operatorname{Diff}^{\text {or }}(N) \longrightarrow \coprod_{N \in \mathcal{M}^{0}(M)} \operatorname{Cob}^{*}(M, N) / \operatorname{Diff}(N)
$$

where "or" stands for "oriented". Now, by [44, 33], $\mathrm{Cob}^{*, \text { or }}(M, N)$ is in bijection with the set of isometries between the intersection forms of $M$ and $N$.

Examples 5.9. The above discussion implies the following facts.

(1) the case $M=S^{4}$. The intersection form is trivial, so $\operatorname{Cob}^{*}$ or $(M, N)$ has one element for each oriented homotopy sphere $N$. Note that $\operatorname{Cob}^{*, o r}(M,-M)$ and $\mathrm{Cob}^{*, o r}(M, M)$ are represented by the mapping cylinders of the identity or a reflection. By Lemma 2.4 these cobordisms both represent $\left[S^{4} \times I\left[\right.\right.$ in $\mathcal{B}\left(S^{4}\right)$. (2) the case $M=\mathbb{C} P^{2}$. The set $\mathrm{Cob}^{*, \text { or }}(M,-M)$ has one element and $\mathrm{Cob}^{*, \text { or }}(M, M)$ is empty.

(3) Results given in [44, Proposition 8 and its proof] imply, for instance, that $\mathrm{Cob}^{* \text { or }}(M, M) / \operatorname{Diff}^{\text {or }}(M)$ is infinite for $M=\mathbb{C} P^{2} \sharp k \overline{\mathbb{C} P^{2}}(k \geq 9)$.

The following result is a direct consequence of Example (1) above.

Proposition 5.10. The set $\mathcal{B}\left(S^{4}\right)$ consists of one element if and only if the smooth Poincaré conjecture is true in dimension 4.

\section{The case $n \leq 3$}

We start with the proof of Theorem C of the introduction (and then Theorem A in low dimensions).

Proof of Theorem C. There is only one closed manifold in dimension 1, namely the circle. Closed surfaces are classified up to diffeomorphism by their fundamental group. This proves Theorem $\mathrm{C}$ when $n \leq 2$.

In dimension 3 , let $M$ and $N$ be closed smooth orientable manifolds. Thanks to to the proof of the geometrization conjecture [52, we know that $M$ and $N$ are geometric in the sense of Thurston. Therefore, if if $M$ and $N$ are h-cobordant, a theorem of Turaev [61, Theorem 1.4] implies that they are homeomorphic, and hence diffeomorphic by smoothing theory [53, Theorem 6.4].

Remark 6.1. Theorem C also follows from a theorem of Kwasik-Schultz which is interesting in itself: an h-cobordism between geometric closed 3-dimensional manifolds $M$ and $N$ is an s-cobordism [38, Theorem p. 736]. One thus get a simple homotopy equivalence from $N$ to $M$, and such a map is homotopic to a diffeomorphm by [62, Theorem 1] or [38, Theorem 1.1]. 
Remark 6.2. We do not know if Theorem $\mathrm{C}$ is true for closed non-orientable manifolds in dimension 3. The proof of [38, Theorem 1.1] uses the splitting theorem for homotopy equivalences of 24 , which is wrong in general for nonorientable manifolds (see [23]). Currently, a positive answer for the simplification problem for closed non-orientable 3-manifolds is only known for $P^{2}$ irreducible ones, i.e. irreducible (every embedded 2 -sphere bounds a 3 -ball) and not containing any 2 -sided $\mathbb{R} P^{2}$. Such manifolds are indeed determined up to diffeomorphism by their fundamental group [22.

We now turn our attention to the set $\mathcal{B}(M)$.

Proposition 6.3. Let $M$ be a smooth closed manifold of dimension $n \leq 2$. Then $\mathcal{B}(M)$ contains one element.

Proof. Let $(W, M, N)$ be an h-cobordism with $n \leq 2$. We claim that $W \approx_{\text {diff }}$ $M \times I$ if $n \leq 2$ (this implies that $W \approx_{\text {diff }} M \times I($ rel $M)$ ). As an invertible cobordism is an h-cobordism by Proposition 3.11, this will prove the proposition. The claim is obvious for $n=0$ and, for $n=1$, it follows from the classification of surfaces with boundary. The case $n=2$ splits into three cases. We shall use the cobordisms $R_{-}=\left(D^{3}, \emptyset, S^{2}\right)$ and $R_{+}=\left(D^{3}, S^{2}, \emptyset\right)$.

(1) $M=S^{2}$. Let $\left(W, S^{2}, N\right)$ be an h-cobordism. By the classification of surfaces, there is a diffeomorphism $h: S^{2} \rightarrow N$ and $\hat{W}=W \circ C_{h}$ is an h-cobordism from $S^{2}$ to itself, with $W \approx_{\text {diff }} \hat{W}\left(\operatorname{rel} S^{2}\right)$. Then, $\Sigma^{3}=R_{-} \circ \hat{W} \circ R_{+}$is a homotopy sphere, which is diffeomorphic to $S^{3}$ by Perelman's theorem. Therefore, $\hat{W}$ is diffeomorphic to $S^{3}$ minus the interior of two smoothly embedded 3-disks, implying that $\hat{W} \approx_{\text {diff }} S^{2} \times I$.

(2) $M=\mathbb{R} P^{2}$. Suppose that $M=\mathbb{R} P^{2}$. By composing $W$ with a mapping cylinder, we may assume that $N=\mathbb{R} P^{2}$. Let $(\tilde{W}, \tilde{M}, \tilde{N})$ be the universal covering of $W$, equipped with its involution $\tau$ (the deck transformation). One has $\tilde{M}=\tilde{N}=S^{2}$, on which $\tau$ is the antipodal involution. As in (1), form the closed 3-manifold $\Sigma^{3}=R_{-}^{3} \circ \hat{W} \circ R_{+}^{3}$, diffeomorphic to $S^{3}$ by Perelman's theorem. The involution $\tau$ extends to an involution $\bar{\tau}$ on $\Sigma$ with two fixed points $p_{ \pm}$. By Part (c) of Proposition 3.5. $W-\partial W \approx_{\text {diff }} M \times \mathbb{R}$. Therefore, $\Sigma-\left\{p_{ \pm}\right\}$is equivariantly diffeomorphic to $S^{2} \times \mathbb{R}$ equipped with the involution $\hat{\tau}(x, t)=(-x, t)$.

Hence, $(\Sigma, \bar{\tau})$ is equivariantly homeomorphic to the suspension of $\left(S^{2}, \tau\right)$. It follows that $\tilde{W}$ is equivariantly homeomorphic to $\left(S^{2} \times I, \hat{\tau}\right)$. Hence, $W \approx_{\text {top }}$ $\mathbb{R} P^{2} \times I$, implying that $W \approx_{\text {diff }} \mathbb{R} P^{2} \times I$.

(3) $\chi(M) \leq 0$. The discussion in [57, pp. 97-99] implies that $W \approx_{\text {diff }} M \times I$.

Much less is known about $\mathcal{B}(M)$ when $M$ is a closed 3-manifold. When $M$ is orientable, we already used (in the proof of Theorem C) the KwasikSchultz result that the Whitehead torsion map $\mathcal{T}: \mathcal{B}(M) \rightarrow \mathrm{Wh}(M)$ is identically zero. However, there are non-trivial s-cobordisms (see e.g. [6, 35] for results and references). The following question seems to be open.

Question 6.4. Is a smooth h-cobordism between closed 3-dimensional invertible? 
Here is a partial answer.

Proposition 6.5. Let $(W, M, N)$ be an s-cobordism between closed manifolds of dimension 3. Suppose that $\pi_{1} M$ is poly-(finite or cyclic). Then, $W$ is topologically invertible with $W^{-1}=\bar{W}$.

Proof. (following [54, Lemma 7.8]). Consider $K=W \times I$ as a cobordism relative boundary from $M \times I$ to $(W \times 0) \circ(N \times I) \circ(\bar{W} \times 1) \approx_{\text {diff }} W \circ \bar{W}(\operatorname{rel} \partial)$. Then $K$ is an s-cobordism. As $\operatorname{dim}(W \times I)=4$ and $\pi_{1} M$ is poly-(finite or cyclic), the topological s-cobordism theorem implies that $W \approx_{\text {diff }}(M \times I) \times I(\operatorname{rel} M \times I \times 0)$. Therefore, $W \circ \bar{W} \approx_{\text {top }} M \times I(\operatorname{rel} M)$. The same argument using the end $N \times I$ of $K$ gives that $\bar{W} \circ W \approx_{\text {top }} N \times I(\operatorname{rel} M)$.

Here are two partial results when $M=S^{3}$.

Proposition 6.6. Let $\left(W, S^{3}, N\right)$ be a smooth h-cobordism. Then $W \approx_{\text {top }} S^{3} \times$ $I\left(\operatorname{rel} S^{3}\right)$.

Proof. It is enough to prove the statement for $W$ a topological h-cobordism. By Perelman's theorem, there is a homeomorphism $h: S^{3} \rightarrow N$ and $\hat{W}=W \circ C_{h}$ is an h-cobordism from $S^{3}$ to itself, with $W \approx_{\text {top }} \hat{W}\left(\operatorname{rel} S^{3}\right)$. As in the proof of Proposition 6.3 (case of $M=S^{2}$ ), this implies that $W$ is the complement of two disjoint tame 4-disks in a homotopy sphere $\Sigma^{4}$. By Freedman's theorem, $\Sigma \approx_{\text {top }} S^{4}$, which implies that $W \approx_{\text {top }} S^{3} \times I\left(\operatorname{rel} S^{3}\right)$.

Corollary 6.7. The following assertions are equivalent.

(a) Any smooth $h$-cobordism $\left(W, S^{3}, N\right)$ is diffeomorphic to $S^{3} \times I$ relative $S^{3}$.

(b) The smooth Poincaré conjecture is true in dimension 4.

Proof. The proof of Proposition 6.6 shows that (b) implies (a). Conversely, let $\Sigma$ be a smooth homotopy 4 -sphere and let $K$ be a smooth submanifold of $\Sigma$ with $K \approx_{\text {diff }} D^{4} \amalg D^{4}$. Then $W=\Sigma-\operatorname{int} K$ is a smooth h-cobordism from $S^{3}$ to $S^{3}$. If (a) is true, then $\Sigma \approx_{\text {diff }} D^{4} \cup_{h} D^{4}$ for some self-diffeomorphism $h$ of $S^{3}$. Therefore, $\Sigma \approx_{\text {diff }} S^{4}[7]$.

We finish with the following open question.

Question 6.8. If $(W, M, N)$ is an h-cobordism with $\operatorname{dim} M=3$, do we have $S^{1} \times W \approx_{\text {diff }}\left(S^{1} \times M\right) \times I\left(\operatorname{rel} S^{1} \times M\right)$ ? Note that the Whitehead torsion will vanish, by the product formula (3.12). Hence this is true if $\operatorname{dim} M \geq 4$.

\section{$7 \quad$ Classifications of $\mathbb{R}$-diffeomorphisms}

In this section we examine the construction in Proposition 3.3 further, aiming for a full classification of $\mathbb{R}$-diffeomorphisms. The diffeomorphisms are classified under three levels of relations: isotopy, decomposability and concordance. 
Let $M$ and $N$ be closed manifolds. Let $\operatorname{Diff}_{\mathbb{R}}(M, N)$ be the set of 1-diffeomorphisms from $N$ to $M$, endowed with the $\mathcal{C}^{\infty}$-topology. Thus, $\pi_{0}\left(\operatorname{Diff}_{\mathbb{R}}(M, N)\right)$ is the set of isotopy classes of such $\mathbb{R}$-diffeomorphisms. For simplicity's sake, we restrict our attention to the subspace $\operatorname{Diff}_{\mathbb{R}}^{+}(M, N)$ of those $\mathbb{R}$-diffeomorphisms $f$ preserving ends, in the sense that $f(N \times[0, \infty)) \subset M \times(r, \infty)$, for some $r \in \mathbb{R}$ (see also Remark 7.3). As in Section 3 . Diff $(N)$ denotes the topological group of self diffeomorphims of $N$.

In the proof of Proposition 3.3 , an invertible cobordism $\left(A_{f}, j_{M}^{r}, f \circ j_{N}^{s}\right)($ for suitable $r$ and $s$ ) was associated to $f \in \operatorname{Diff}_{\mathbb{R}}(N, M)$. Consider its class $\mathcal{A}_{f}$ in $\operatorname{Cob}^{*}(M, N)$. Here is the fundamental observation leading to the other classification results. It is valid in all dimensions.

Theorem 7.1. The correspondence $f \mapsto\left(A_{f}, j_{M}^{r}, f \circ j_{N}^{s}\right)$ induces a bijection

$$
\mathcal{A}: \pi_{0}\left(\operatorname{Diff}_{\mathbb{R}}^{+}(M, N)\right) \stackrel{\approx}{\longrightarrow} \operatorname{Cob}^{*}(M, N) .
$$

Moreover, $\mathcal{A}\left(\operatorname{id}_{M \times \mathbb{R}}\right)=\mathbf{1}_{M}$, and if $f \in \operatorname{Diff}_{\mathbb{R}}^{+}(N, M)$ and $g \in \operatorname{Diff}_{\mathbb{R}}^{+}(P, N)$, then $\mathcal{A}(f \circ g)=\mathcal{A}(f) \circ \mathcal{A}(g)$.

Before we proceed, we remark that this gives a new interpretation of the category of invertible cobordisms.

Corollary 7.2. The category $\mathrm{Cob}^{*}$ is isomorphic to the opposite of the category where the objects are smooth manifolds and the set of morphisms from $M$ to $N$ is $\pi_{0}\left(\operatorname{Diff}_{\mathbb{R}}^{+}(M, N)\right)$.

Proof of Theorem 7.1. The proof involves several steps.

(1) $\mathcal{A}$ is well defined. Let $f: N \times \mathbb{R} \rightarrow M \times \mathbb{R}$ be an element of $\operatorname{Diff}_{\mathbb{R}}^{+}(N, M)$. We use the notations of the proof of Proposition 3.3. $M_{r}=M \times\{r\}, N_{u}=N \times\{u\}$, $N_{u}^{\prime}=f\left(N_{u}\right)$, etc. Recall that, to define $A_{f}$, we choose $u$ and $r<s$ in $\mathbb{R}$ such that $N_{u}^{\prime} \subset M \times(r, s)$. The region from $M_{r}$ to $N_{u}^{\prime}$ constitutes $A_{f}$ and that between $N_{u}^{\prime}$ and $M_{s}$ constitutes the inverse $B_{f}$ of $A_{f}$. It is easy to check that $\left[A_{f}\right]=\left[A_{f}, j_{M}^{r}, f \circ j_{N}^{u}\right] \in \operatorname{Cob}^{*}(M, N)$ does not depend on the choices of $r$ and $u$. Consequently, we may assume that $u=0$.

Let $f_{t}: N \times \mathbb{R} \rightarrow M \times \mathbb{R}(t \in I)$ be an isotopy between $f_{0}=f$ and $f_{1}=\hat{f}$. Let $g_{t}$ be the restriction of $f_{t}$ to $N_{0}$. Since $N$ is compact, there exist $r<r_{1}<s_{1}<s$ in $\mathbb{R}$ such that $g_{t}\left(N_{0}\right) \subset M \times\left(r_{1}, s_{1}\right)$ for all $t$. By the isotopy extension theorem on $M \times[r, s]$ [26. Theorem 1.3 in Chapter 8], there exists an ambient isotopy $F_{t}: M \times \mathbb{R} \rightarrow M \times \mathbb{R}$, which is the identity outside $M \times\left[r_{1}, s_{1}\right]$ and such that $g_{t}=F_{t} \circ g_{0}$. Using $r$ to define both $A_{f_{0}}$ and $A_{f_{1}}$, we see that $F_{1}$ provides a diffeomorphism from $A_{f}$ to $A_{\hat{f}}$ (relative $M_{r}$ ) such that $F_{1} \circ f \circ j_{N}^{0}=\hat{f} \circ j_{N}^{0}$. Therefore, $\left[A_{f}\right]=\left[A_{\hat{f}}\right]$ in $\operatorname{Cob}^{*}(M, N)$.

(2) $\mathcal{A}$ is surjective. Let $A=\left(A, j_{M}, j_{N}\right)$ represent a class $\alpha \in \operatorname{Cob}^{*}(M, N)$ and let $B=A^{-1}$. Composing infinitely many copies of $A \circ B$ as in (3.7), we obtain a manifold $W$ together with two diffeomorphisms

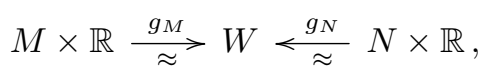


Then $h=g_{M}^{-1} \circ g_{N}: N \times \mathbb{R} \rightarrow M \times \mathbb{R}$ is an element of $\operatorname{Diff}_{\mathbb{R}}^{+}(N, M)$ such that $\left[A_{h}\right]=[A]$. Hence, $\mathcal{A}(h)=\alpha$.

(3) $\mathcal{A}$ is injective. Let $f$ and $\hat{f}$ in $\operatorname{Diff}_{\mathbb{R}}^{+}(M, N)$ such that $\mathcal{A}(f)=\mathcal{A}(\hat{f})$. Using observations in (1), we can represent $\mathcal{A}(f)$ by $\left(A_{f}, j_{M}^{0}, f \circ j_{N}^{u}\right)$ and $\mathcal{A}(\hat{f})$ by $\left(A_{\hat{f}}, j_{M}^{0}, \hat{f} \circ j_{N}^{\hat{u}}\right)$, where we may assume that $N_{u}^{\prime} \subset \operatorname{int} A_{\hat{f}}$. In fact, after suitable isotopies of $f$ and $\hat{f}$ (by translations in the $\mathbb{R}$-direction) we may even assume that $u=\hat{u}=0$. This means that we can write $\left[A_{\hat{f}}\right]=\left[A_{f}\right] \circ[K]$, where $[K]=\left[K, f \circ j_{N}^{0}, \hat{f} \circ j_{N}^{0}\right]$. But if $\mathcal{A}(f)=\mathcal{A}(\hat{f})$, the invertible cobordism $K$ must be equivalent to $\mathbf{1}_{N}$, i. e. there exists a diffeomorphsim $F: N \times I \rightarrow K$ such that $F(x, 0)=f(x, 0)$ and $F(x, 1)=\hat{f}(x, 0)$ for all $x \in N$.

Now think of $F$ as an isotopy of embeddings from $f \circ j_{N}^{0}$ to $\hat{f}_{\circ} j_{N}^{0}$. By the isotopy extension theorem there exists an ambient isotopy $H_{t}$ of $M \times \mathbb{R}$ such that $H_{0}=\operatorname{id}_{M \times \mathbb{R}}$ and $\left.H_{1} \circ f(x, 0)\right)=\hat{f}(x, 0)$ for all $x \in N$.

Define $G: N \times \mathbb{R} \rightarrow N \times \mathbb{R}$ by $G=\hat{f}^{-1} \circ H_{1} \circ f$. Then $G$ is a diffeomorphism such that $G(x, 0)=(x, 0)$ for all $x \in N$. Considering $G$ and $\operatorname{id}_{N \times \mathbb{R}}$ as tubular neighborhoods of $N \times\{0\}$ in $N \times \mathbb{R}$, we see that $G$ is isotopic to the identity, by uniqueness [26, Theorem 5.3 in Chapter 4]. It follows that $\hat{f}$ is isotopic to $\hat{f} \circ G=H_{1} \circ f$, hence also to $H_{0} \circ f=f$.

(4) It is obvious that $\mathcal{A}\left(\operatorname{id}_{M \times \mathbb{R}}\right)=\mathbf{1}_{M}$, and it remains to prove the composition formula. Let $f \in \operatorname{Diff}_{\mathbb{R}}^{+}(N, M)$ and $g \in \operatorname{Diff}_{\mathbb{R}}^{+}(P, N)$. Start by choosing $u \in \mathbb{R}$ such that $f\left(N_{u}\right) \subset M \times(0, \infty)$, and then $v \in \mathbb{R}$ such that $g\left(P_{v}\right) \subset N \times(u, \infty)$. Then the regions $A_{g}$ between $N_{u}$ and $g\left(P_{v}\right), A_{f}$ between $M_{0}$ and $f\left(N_{u}\right)$, and $A_{f \circ g}$ between $M_{0}$ and $f \circ g\left(P_{v}\right)$ can be used to define $\mathcal{A}(g), \mathcal{A}(f)$ and $\mathcal{A}(f \circ g)$, respectively. In other words,

$$
\begin{aligned}
\mathcal{A}(g) & =\left[A_{g}, j_{N}^{u}, g \circ j_{P}^{v}\right] \\
\mathcal{A}(f) & =\left[A_{f}, j_{M}^{0}, f \circ j_{N}^{u}\right] \\
\mathcal{A}(f \circ g) & =\left[A_{f \circ g}, j_{M}^{0}, f \circ g \circ j_{P}\right]
\end{aligned}
$$

Now observe that we can write $A_{f \circ g}$ as $A_{f} \cup f\left(A_{g}\right)$, and consequently

$$
\begin{aligned}
{\left[A_{f \circ g}, j_{M}^{0}, f \circ g \circ j_{P}\right] } & =\left[A_{f}, j_{M}^{0}, f \circ j_{N}^{u}\right] \circ\left[f\left(A_{g}\right), f \circ j_{N}^{u}, f \circ g \circ j_{P}\right] \\
& =\left[A_{f}, j_{M}^{0}, f \circ j_{N}^{u}\right] \circ\left[A_{g}, j_{N}^{u}, g \circ j_{P}\right] \\
& =\mathcal{A}_{f} \circ \mathcal{A}_{g}
\end{aligned}
$$

We are now interested in another equivalence relation amongst $\mathbb{R}$-diffeomorphism, using decomposability. A $\mathbb{R}$-diffeomorphism $f \in \operatorname{Diff}_{\mathbb{R}}^{+}\left(Q, Q^{\prime}\right)$ is called decomposable if there exists a diffeomorphism $\varphi: Q^{\prime} \rightarrow Q$ such that $f$ is isotopic to $\varphi \times \operatorname{id}_{\mathbb{R}}$. Fix a manifold $M$ and consider pairs $(N, f)$ where $N$ is a smooth closed manifold and $f: N \times \mathbb{R} \rightarrow M \times \mathbb{R}$ is a diffeomorphism. Two such pairs $(N, f)$ and $(\hat{N}, \hat{f})$ are equivalent (notation: $(N, f) \sim(\hat{N}, \hat{f}))$ if $f^{-1} \circ \hat{f}$ is decomposable. The set of equivalences classes is denoted by $\mathcal{D}(M)$. Note that $(N, f)$ is decomposable if and only if $(N, f) \sim(M$, id). 
Remark 7.3. The above definition of $\mathcal{D}(M)$ is equivalent to the one presented in the introduction, where $\mathbb{R}$-diffeomorphisms were not supposed to preserve ends. Indeed, $\operatorname{Diff}_{\mathbb{R}}^{+}(M, N)$ is a fundamental domain for the action of $\{ \pm 1\} \approx$ $\left\{\operatorname{id}_{N} \times \pm \operatorname{id}_{\mathbb{R}}\right\}$ by precomposition.

Theorem 7.4. Let $M$ be a smooth closed manifold. The correspondence $(N, f) \mapsto$ $\left[A_{f}[\right.$ induces a bijection

$$
B: \mathcal{D}(M) \stackrel{\approx}{\longrightarrow} \mathcal{B}(M) .
$$

Moreover, $B(N, f)=[M \times I$ if and only if $f$ is decomposable.

Proof. Actually, the map $B$ is induced from the bijection $\mathcal{A}$ of Theorem 7.1. As in Lemma 3.8, let $\mathcal{M}_{n}^{0}$ be a set of representatives of the diffeomorphism classes of closed manifolds of dimensioni $n$. Consider the commutative diagram

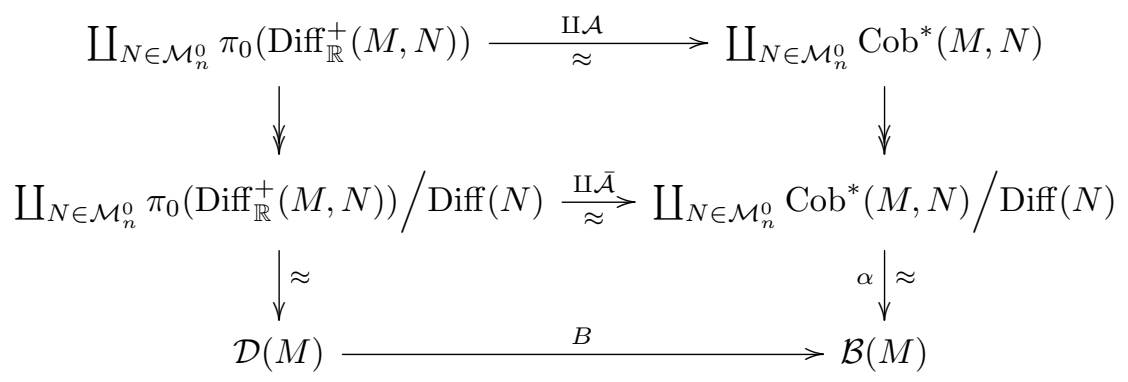

The map $\amalg \mathcal{A}$ is a bijection by Theorem 7.1. It intertwines the right-actions of $\operatorname{Diff}(M)$ on $\operatorname{Cob}^{*}(M, N)$ of Lemma 3.8 with the ones defined on $\pi_{0}\left(\operatorname{Diff}_{\mathbb{R}}^{+}(M, N)\right)$ by pre-composition using the inclusion $\operatorname{Diff}(N) \rightarrow \operatorname{Diff}_{\mathbb{R}}^{+}(N, N)$ given by $\varphi \mapsto$ $\varphi \times \operatorname{id}_{\mathbb{R}}$. The latter corresponds to the equivalence relation $\sim$ (note that $N \approx_{\text {diff }}$ $\hat{N}$ if $(N, f) \sim(\hat{N}, \hat{f}))$. That the map $\alpha$ is a bijection is the statement of Lemma 3.8. Thus, the map $B$ is bijective.

Remark 7.5. From Part (2) of the proof of Theorem 7.1, it follows that $(N, f) \sim\left(N, g_{M}^{-1} \circ g_{N}\right)$, where $g_{M}$ and $g_{N}$ are the diffeomorphisms constructed in in 7.20 .

Thanks to Proposition 6.3. Proposition 5.10 and Corollary 6.7. Theorem 7.4 admits the following corollary.

Corollary 7.6. Any diffeomorphism $f: N \times \mathbb{R} \rightarrow M \times \mathbb{R}$ is decomposable if $\operatorname{dim} M \leq 2$. When $N=M=S^{n}$ with $n=3,4$, this is true if and only if the smooth Poincaré conjecture is true in dimension 4.

The bijection $B: \mathcal{D}(M) \rightarrow \mathcal{B}(M)$ of Theorem 7.4 may be composed with the map $\mathcal{T}: \mathcal{B}(M) \rightarrow \mathrm{Wh}(M)$, associating to $W$ its Whitehead torsion $\tau(W, M)$. This gives a map $T: \mathcal{D}(M) \rightarrow \mathrm{Wh}(M)$. By Theorem 3.15, $\mathcal{T}$ is a bijection when $n \geq 5$. Thus, Theorem 7.4 has the following corollary. 
Corollary 7.7. Let $M$ be a smooth closed manifold of dimension $\geq 5$. Then, the map $T: \mathcal{D}(M) \rightarrow \mathrm{Wh}(M)$ is a bijection. Moreover, $T(N, f)=0$ if and only if $f$ is decomposable.

Corollary 7.7 implies Theorem D and Corollary E of the introduction. Another immediate consequence is the following:

Corollary 7.8. Let $M$ be a closed manifold and let $K$ be a closed manifold with Euler characteristic 0. The map $\mathcal{D}(M) \rightarrow \mathcal{D}(M \times K)$ given by product with the identity map on $K$ is trivial.

In other words: if $f: N \times \mathbb{R} \underset{\rightarrow}{\widetilde{\sim}} M \times \mathbb{R}$ is a diffeomorphism, then $f \times \mathrm{id}_{K}$ is isotopic to a diffeomorphism of the form $h \times \operatorname{id}_{\mathbb{R}}$, where $h$ is a diffeomorphism $N \times K \rightarrow M \times K$.

Proof. The bijections $\mathcal{D}(M) \approx \mathcal{B}(M) \approx \mathrm{Wh}(M)$ commute with product with $K$. The result then follows by the product formula for Whitehead torsion 3.12).

Diagram (7.21) gives a partition of $\mathcal{D}(M)$ indexed by diffeomorphism classes of manifolds. Particularly interesting is the class corresponding to $M$ itself, which via the bijection $\mathcal{B}$ corresponds to the inertial cobordisms:

$$
\mathcal{I B}(M)=\operatorname{Cob}^{*}(M, M) / \operatorname{Diff}(M) \approx \pi_{0}\left(\operatorname{Diff}_{+}(M \times \mathbb{R})\right) / \operatorname{Diff}(M) .
$$

Corollary 7.9. Let $M$ be a smooth closed manifold. The following assertions are equivalent.

(a) Any automorphism $g: M \times \mathbb{R} \rightarrow M \times \mathbb{R}$ is decomposable.

(b) $\mathcal{I B}(M)$ has one element.

Moreover, if $\operatorname{dim} M \geq 5$, Assertion (b) may be replaced by

(b') $I(M)=\{0\}$.

Manifolds $M$ such that $I(M)=\{0\}$ may be found in Example 4.5 .

Example 7.10. Given two diffeomorphisms $f, g: N \times \mathbb{R} \rightarrow M \times \mathbb{R}$, it is possible that $f^{-1} \circ g$ is decomposable but not $g \circ f^{-1}$. An example of this sort may be obtained using Corollary 7.9 and Part (3) of Example 4.5 .

In formula 7.22 the second action is right multiplication by the image of the group homomorphism $\pi_{0}(\operatorname{Diff}(M)) \rightarrow \pi_{0}\left(\operatorname{Diff}_{\mathbb{R}}^{+}(M)\right)$ induced by $\varphi \mapsto$ $\varphi \times \operatorname{id}_{\mathbb{R}}$, and this corresponds to the map (also homomorphism!) $\pi_{0}(\operatorname{Diff}(M)) \rightarrow$ $\operatorname{Cob}^{*}(M, M)$ given by $f \mapsto C_{f^{-1}}$ (mapping cylinder). As seen in Example 2.5 . this map is not injective, but has as kernel the isotopy classes of diffeomorphisms concordant to the identity. This leads to the following result, first proved by W. Ling in the topological category [45]. Let $C(M)=\{f \in \operatorname{Diff}(M \times I)|f| M \times$ $\{0\}=\mathrm{id}\}$ be the space of concordances of $M$. Then evaluation on $M \times\{1\}$ gives rise to a fibration (over a union of components) $C(M) \rightarrow \operatorname{Diff}(M)$, with fiber $\operatorname{Diff}(M \times I$, rel $M \times \partial I)$. 
Proposition 7.11. The long, exact sequence of homotopy groups of this fibration ends as follows:

$$
\cdots \longrightarrow \pi_{0}(C(M)) \longrightarrow \pi_{0}(\operatorname{Diff}(M)) \longrightarrow \pi_{0}\left(\operatorname{Diff}_{\mathbb{R}}^{+}(M)\right) \longrightarrow \mathcal{I B}(M)
$$

Proof. The last map in the ordinary long exact sequence is the homomorphism $\pi_{0}(C(M)) \rightarrow \pi_{0}(\operatorname{Diff}(M))$ with image the set of isotopy classes of diffeomorphisms concordant to the identity, which we just saw is also the kernel of the homomorphism $\pi_{0}(\operatorname{Diff}(M)) \rightarrow \pi_{0}\left(\operatorname{Diff}_{\mathbb{R}}^{+}(M)\right)$. The last map is just the quotient map onto the set of left cosets.

Remark 7.12. It is known that $\operatorname{Diff}(M \times \mathbb{R})$ is a non-connected delooping of $\operatorname{Diff}(M \times I$, rel $M \times \partial I)$. (See e. g. 68.) Proposition 7.11 gives more information on components.

We now use the relation of concordance to give a classification of 1-diffeomorphisms which is coarser than isotopy. Following the pattern above, we first say that a $\mathbb{R}$-diffeomorphism $f \in \operatorname{Diff}_{\mathbb{R}}^{+}\left(Q^{\prime}, Q\right)$ is c-decomposable if there exists a diffeomorphism $\varphi: Q^{\prime} \rightarrow Q$ such that $f$ is concordant to $\varphi \times i d_{\mathbb{R}}$. Then $(\hat{N}, \hat{f})$ and $(N, f)$ are called c-equivalent (notation: $\left.(\hat{N}, \hat{f}) \sim_{c}(N, f)\right)$ if $f^{-1} \circ \hat{f}$ is cdecomposable. Of course, $(\hat{N}, \hat{f}) \sim(N, f)$ implies $(\hat{N}, \hat{f}) \sim_{c}(N, f)$; therefore, the set $\mathcal{D}_{c}(M)$ of these c-equivalences classes is a quotient of $\mathcal{D}(M)$.

Using the the bijection $B$ of Theorem 7.4 the equivalence relation $\sim_{c}$ on $\mathcal{D}(M)$ may be transported to $\mathcal{B}(M)$, giving rise to an equivalence relation on $\mathcal{B}(M)$, also denoted $\sim_{c}$. We want to prove that $\sim_{c}$ can be described in terms of the relation of concordance of invertible cobordisms, defined in Remark 3.17 .

Recall again the partition

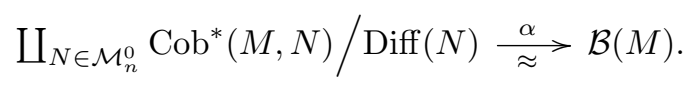

of Lemma 3.8. In Remark 3.17 the relation of (invertible) concordance is defined on each set $\operatorname{Cob}^{*}(M, N)$, and the action of $\operatorname{Diff}(N)$ descends to the set of concordance classes $\overline{\mathrm{Cob}^{*}}(M, N)$. Set

$$
\mathcal{B}_{c}(M)=\coprod_{N \in \mathcal{M}_{n}^{0}} \overline{\operatorname{Cob}^{*}}(M, N) / \operatorname{Diff}(N)
$$

Like Theorem 7.4, the following result is valid in all dimensions.

Theorem 7.13. Let $M$ be a smooth closed manifold. Then, the bijection $B: \mathcal{D}(M) \rightarrow \mathcal{B}(M)$ of Theorem 7.4 descends to a bijection

$$
B_{c}: \mathcal{D}_{c}(M) \stackrel{\approx}{\longrightarrow} \mathcal{B}_{c}(M) .
$$


Proof. Given Part (i) of the proof of Theorem 7.4, in order to define $B_{c}$, we just need to prove that when $f, \hat{f}: N \times \mathbb{R} \rightarrow M \times \mathbb{R}$ are concordant, then $\left[A_{f}\right]=\left[A_{f}, j_{M}^{r} \amalg f \circ j_{N}^{0}\right]$ and $\left[A_{\hat{f}}\right]=\left[A_{\hat{f}}, j_{M}^{r} \amalg \hat{f} \circ j_{N}^{0}\right]$ represent the same class in $\overline{\operatorname{Cob}^{*}(M, N)}$. Let $F: I \times N \times \mathbb{R} \rightarrow I \times M \times \mathbb{R}$ be a concordance between $f$ and $\hat{f}$. The construction of $A_{f}, B_{f}, A_{\hat{f}}$ and $B_{\hat{f}}$ may be done globally in $I \times N$ and $I \times M$. This would provide cobordisms $A_{F}$ between $A_{f}, A_{\hat{f}}$, and $B_{F}$ between $B_{f}, B_{\hat{f}}$ which are inverse of one another, which is what we need.

The map $B_{c}$ is thus well defined. It is surjective, since $B$ is. To prove that $B_{c}$ is injective, we use a relative version of the proof of surjectivity in Theorem 7.1 . Let $(N, f)$ and $(\hat{N}, \hat{f})$ represent classes in $\mathcal{D}(M)$ such that $B(N, f) \sim_{c} B(\hat{N}, f)$. Since the relation $\sim_{c}$ preserves $\operatorname{Cob}^{*}(M, N)$, this means that there is a diffeomorphism $\gamma: \hat{N} \rightarrow N$ such that $B(\hat{N}, \hat{f}) \sim B\left(N, \hat{f}_{\circ}\left(\gamma \times \operatorname{id}_{\mathbb{R}}\right)\right)$. This permits us to assume that $\hat{N}=N$. In this case, $B(N, f)$ and $B(N, \hat{f})$ are represented by $\left[A_{f}\right]$ and $\left[A_{\hat{f}}\right]$ in $\operatorname{Cob}^{*}(M, N)$ such that $\left[A_{f}\right]$ is invertibly concordant to $\left[A_{\hat{f}}\right] \beta$ for some $\beta \in \operatorname{Diff}(N)$. Using again that $(N, \hat{f}) \sim\left(N, \hat{f} \circ\left(\beta \times \operatorname{id}_{\mathbb{R}}\right)\right)$, we may assume that $\left[A_{\hat{f}}\right]=\left[A_{f}\right]$ in $\overline{\operatorname{Cob}^{*}}(M, N)$.

Let $[K]$ be a concordance between $A_{f}$ and $A_{\hat{f}}$, with inverse $[L]$ from $\left[B_{f}\right]$ and $\left[B_{\hat{f}}\right]$. Let $K_{i}$ and $L_{i}(i \in \mathbb{Z})$ be copies of $K$ and $L$. As in (3.7), we form the manifold

$$
\begin{aligned}
X & =\cdots \circ\left(K_{i} \circ L_{i}\right) \circ\left(K_{i+1} \circ L_{i+1}\right) \circ \cdots \\
& =\cdots \circ\left(L_{i} \circ K_{i+1}\right) \circ\left(L_{i+1} \circ K_{i+2}\right) \circ \cdots
\end{aligned}
$$

Using convenient diffeomorphisms $K_{i} \circ L_{i} \approx_{\text {diff }} I \times M \times I$ and $L_{i} \circ K_{i+1} \approx_{\text {diff }}$ $I \times N \times I$, one gets, as in 7.20 , two diffeomorphisms

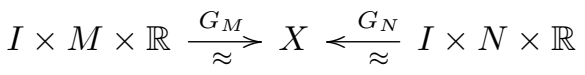

The diffeomorphism $F=G_{M}^{-1} \circ G_{N}: I \times N \times \mathbb{R} \rightarrow I \times M \times \mathbb{R}$ restricts to diffeomorphisms $F_{i}:\{i\} \times N \times \mathbb{R} \rightarrow\{i\} \times M \times \mathbb{R}(i=0,1)$ and $F$ constitutes a concordance between $F_{0}$ and $F_{1}$. Therefore, $\left(N, F_{0}\right) \sim_{c}\left(N, F_{1}\right)$. By Remark 7.5 one has $\left(\{0\} \times N, F_{0}\right) \sim(N, f)$ and $\left(\{1\} \times N, F_{1}\right) \sim(N, \hat{f})$. Therefore, $(N, f) \sim_{c}$ $(N, \hat{f})$, which proves the injectivity of $B_{c}$.

We now compute $\mathcal{B}_{c}(M)$ when $\operatorname{dim} M \geq 5$, using the bijection $\mathcal{T}: \mathcal{B}(M) \rightarrow$ $\mathrm{Wh}(M)$ of Theorem 3.15. As in Example 4.5, we consider the subgroup $\mathcal{N}(M)$ of $\mathrm{Wh}(M)$ defined by

$$
\mathcal{N}(M)=\left\{\tau+(-1)^{n} \bar{\tau} \mid \tau \in \mathrm{Wh}(M)\right\},
$$

using the involution $\tau \mapsto \bar{\tau}$ of 3.14 .

The following result now follows easily from the discussion at the end of Section 3.

Proposition 7.14. Let $M$ be a smooth closed manifold of dimension $n \geq 5$. Then, the bijection $\mathcal{T}: \mathcal{B}(M) \rightarrow \mathrm{Wh}(M)$ of Theorem 3.15 descends to a bijection

$$
\mathcal{T}_{c}: \mathcal{B}_{c}(M) \stackrel{\approx}{\longrightarrow} \mathrm{Wh}(M) / \mathcal{N}(M) \text {. }
$$


Proof. That $\mathcal{T}_{c}$ is well-defined follows from Lemma 3.18, and surjectivity is trivial. Assume now that the torsions of two invertible cobordisms $\left(W, j_{M}, j_{N}\right)$ and $\left(W^{\prime}, j_{M}^{\prime}, j_{N}^{\prime}\right)$ satisfy the equation $\tau\left(W^{\prime}, j_{M}^{\prime}\right)-\tau\left(W, j_{M}\right)=\sigma+(-1)^{n} \bar{\sigma}$ for some $\sigma \in \operatorname{Wh}(M)$, where $n=\operatorname{dim} M$.

There is a relative h-cobordism $(X, W, V)$ with $\tau(X, W)=j_{M *}(\sigma)$, where $V$ is another h-cobordism from $j_{M}(M)$ to $j_{N}(N)$. By Proposition $3.11 X$ and $V$ are both invertible, and by Lemma 3.18 we have $\tau\left(V, j_{M}\right)=\tau\left(W^{\prime}, j_{M}\right)$. By uniqueness of Whitehead torsion, $\left[W, j_{M}\left[=\left[V, j_{M}[\in \mathcal{B}(M)\right.\right.\right.$.

Theorem 7.13 together with Proposition 7.14 implies the following corollary.

Corollary 7.15. Let $M$ be a smooth closed manifold of dimension $\geq 5$. Then, the bijection $T: \mathcal{D}(M) \rightarrow \mathrm{Wh}(M)$ of Corollary 7.7 descends to a bijection

$$
T_{c}: \mathcal{D}_{c}(M) \rightarrow \mathrm{Wh}(M) / \mathcal{N}(M) .
$$

Moreover, $T_{c}(N, f)=0$ if and only if $f$ is c-decomposable.

Recall the inclusion $\mathcal{N}(M) \subset I(M)$, which is not an equality in general. Corollary 7.15 implies the following result.

Corollary 7.16. Let $M$ be a smooth closed connected manifold of dimension $\geq 5$. The following assertions are equivalent.

(a) Any automorphism $g: M \times \mathbb{R} \rightarrow M \times \mathbb{R}$ is c-decomposable.

(b) $\mathcal{N}(M)=I(M)$.

Example 7.17. Let $M$ be a smooth closed connected manifold of dimension $n \geq 5$ such that $\pi=\pi_{1}(M)$ is cyclic of order 5 with generator $t$. Then, $\mathrm{Wh}(M) \approx \mathbb{Z}$ generated by $\sigma=\left(1-t-t^{4}\right) \in G L_{1}(\mathbb{Z} \pi)$ [50, Example 6.6]. We see that $\sigma=\bar{\sigma}$, so the involution on $\mathrm{Wh}(M)$ is trivial. Therefore,

- if $n$ is odd, $\mathcal{N}(M)=0$ and then $\mathcal{D}(M)=\mathcal{D}_{c}(M) \approx \mathbb{Z}$; thus concordance implies isotopy for $\mathbb{R}$-diffeomorphisms with range $M \times \mathbb{R}$;

- if $n$ is even, then $\mathcal{D}(M) \approx \mathbb{Z}$ and $\mathcal{D}_{c}(M) \approx \mathbb{Z}_{2}$. Thus, for diffeomorphisms with range $M \times \mathbb{R}$, there are infinitely many isotopy classes within the same concordance class.

\section{Miscellaneous}

8.1. This paper deals with $\mathbb{R}$-diffeomorphisms between closed manifolds. For open manifolds, there is a long story of negative answers to the $\mathbb{R}$-simplification problem, starting with the earlier example of J.H.C. Whitehead [70, p. 827]. There is also the famous Whitehead manifold which is $\mathbb{R}$-diffeomorphic but not homeomorphic to $\mathbb{R}^{3}$ (see, e.g. [11, pp. 61-67]). The most striking example is given by the uncountable family of fake $\mathbb{R}^{4}$ 's (see e.g. [16]), which are all $\mathbb{R}$ diffeomorphic, since there is only one smooth structure on $\mathbb{R}^{5}[58$, Corollary 2$]$. 
8.2. Historical note. As seen in Sections 3 , Theorem A of the introduction is equivalent to the smooth h-cobordism theorem of Smale [56] for $n \geq 5$, and to the topological h-cobordism theorem of Freedman for $n=4$ [14]. For $n=3$ it is a consequence of Perelman's proof of the Poincaré conjecture see [51. There is no known proof not using these formidable results for which three Field medals were awarded. Finally, for $n=2$, Theorem A requires the classification of surfaces, a classical but not trivial result. Note that the simplification problem is a geometric form of the problem of recognizing the diffeomorphism type of a smooth closed manifold by its homotopy type, one of the most important problems of algebraic topology, going back to the birth of the subject (see e.g. [20, §5.1].

8.3. $\mathbb{R}^{k}$-diffeomorphisms were introduced by B. Mazur 47] under the name of $k$ equivalences. Note that a diffeomorphism $f: M \times \mathbb{R}^{k} \rightarrow N \times \mathbb{R}^{k}$ induces a stable tangential homotopy equivalence (still called $f$ ) from $M$ to $N$. The thickness of such a stable tangential homotopy equivalence $f$ is the minimal $k$ for which $f$ is induced by an $\mathbb{R}^{k}$-diffeomorphism [41]. This thickness is $\leq \operatorname{dim} M+2$ [4], Theorem 1]. For more results, see e.g. [41, 28, 36].

8.4. The $P$-simplification problem has been studied for $P$ a sphere, a torus or a surface. See e.g. 25] for results and several references, and also Remark 3.4 . For more recent results, see e.g. [36, 28, 40, 37.

8.5. Stable diffeomorphisms. Two closed manifolds $M, N$ of dimension $2 n$ are called stably diffeomorphic in the literature if $M \sharp p\left(S^{n} \times S^{n}\right) \approx_{\text {diff }} N \sharp p\left(S^{n} \times\right.$ $S^{n}$ ) for some integer p. Thus Corollary 4.3 and Proposition 5.6 say that $\mathbb{R}$-diffeomorphism implies stable diffeomorphism. The stable diffeomorphism class of a manifold may be detected by cobordisms invariants, as initiated by M. Kreck 34. For recent results and many references, see 31.

8.6. Generalized spherical spaceforms. A manifold is a generalized spherical spaceform if its universal covering is a homotopy sphere. Let $M$ and $N$ be diffeomorphic generalized spherical spaceforms of dimension $\geq 5$. Then Kwasik and Schultz have proved that any h-cobordism between $M$ and $N$ is trivial 39. This implies that $I(M)=0$ and, thus, $\mathbb{R}$-diffeomorphism implies diffeomorphism.

8.7. In general relativity, the $\mathbb{R}$-simplification problem has natural applications to the classification of Cauchy surfaces in globally hyperbolic spacetimes. (See 60] for results and references).

\section{References}

[1] A. Bak. The involution on Whitehead torsion. General Topology and Appl., 7(2):201206, 1977. NSF-CBMS Regional Conference in Topology (Univ. Tennessee, Knoxville, Tenn., 1974).

[2] A. Bartels, W. Lück, and H. Reich. On the Farrell-Jones conjecture and its applications. arXiv:math/0703548.

[3] H. Bass. The stable structure of quite general linear groups. Bull. Amer. Math. Soc., 70:429-433, 1964. 
[4] A. Borel and J.-P. Serre. Corners and arithmetic groups. Comment. Math. Helv., 48:436491, 1973. Avec un appendice: Arrondissement des variétés à coins, par A. Douady et L. Hérault.

[5] K. S. Brown. Cohomology of groups, volume 87 of Graduate Texts in Mathematics. Springer-Verlag, New York-Berlin, 1982.

[6] S. Cappell and J. Shaneson. On four-dimensional s-cobordisms. II. Comment. Math. Helv., 64(2):338-347, 1989.

[7] J. Cerf. Sur les difféomorphismes de la sphère de dimension trois $\left(\Gamma_{4}=0\right)$. Lecture Notes in Mathematics, No. 53. Springer-Verlag, Berlin-New York, 1968.

[8] L. S. Charlap. Compact flat riemannian manifolds. I. Ann. of Math. (2), 81:15-30, 1965.

[9] M. M. Cohen. A course in simple-homotopy theory. Springer-Verlag, New York-Berlin, 1973. Graduate Texts in Mathematics, Vol. 10.

[10] P. Conner and F. Raymond. Derived actions. In Proc. 2nd Conf. on Compact Transformation Groups (Amherst, MA,1971), Part II, pages 237-310. Lecture Notes in Mathematics, No. 299. Springer Verlag, Berlin-New York, 1972.

[11] G. de Rham. Lectures on introduction to algebraic topology. Tata Institute of Fundamental Research, Bombay, 1969. Notes by V. J. Lal, Tata Institute of Fundamental Research Lectures on Mathematics, No. 44.

[12] F. T. Farrell and W. C. Hsiang. The Whitehead group of poly-(finite or cyclic) groups. J. London Math. Soc. (2), 24(2):308-324, 1981.

[13] R. Fintushel and R. Stern. Six lectures on four 4-manifolds. In Low dimensional topology, volume 15 of IAS/Park City Math. Ser., pages 265-315. Amer. Math. Soc., Providence, RI, 2009.

[14] M. H. Freedman and F. Quinn. Topology of 4-manifolds, volume 39 of Princeton Mathematical Series. Princeton University Press, Princeton, NJ, 1990.

[15] M. H. Freedman and P. Teichner. 4-manifold topology. I. Subexponential groups. Invent. Math., 122(3):509-529, 1995.

[16] R. Gompf. An exotic menagerie. J. Differential Geom., 37(1):199-223, 1993.

[17] A. Hatcher and T. Lawson. Stability theorems for "concordance implies isotopy" and " $h$-cobordism implies diffeomorphism". Duke Math. J., 43(3):555-560, 1976.

[18] J-C. Hausmann. $h$-cobordismes entre variétés homéomorphes. Comment. Math. Helv., 50:9-13, 1975.

[19] J-C. Hausmann. Open books and h-cobordisms. Comment. Math. Helv., 55(3):330-346, 1980.

[20] J-C. Hausmann. Mod two homology and cohomology. Universitext. Springer, Cham, 2014.

[21] J-C Hausmann and P. Vogel. The plus construction and lifting maps from manifolds. In Algebraic and geometric topology (Proc. Sympos. Pure Math., Stanford Univ., Stanford, Calif., 1976), Part 1, Proc. Sympos. Pure Math., XXXII, pages 67-76. Amer. Math. Soc., Providence, R.I., 1978.

[22] W. Heil. On $P^{2}$-irreducible 3-manifolds. Bull. Amer. Math. Soc., 75:772-775, 1969.

[23] H. Hendriks. Une obstruction pour scinder une équivalence d'homotopie en dimension 3. Ann. Sci. École Norm. Sup. (4), 9(3):437-467, 1976.

[24] H. Hendriks and F. Laudenbach. Scindement d'une équivalence d'homotopie en dimension 3. Ann. Sci. École Norm. Sup. (4), 7:203-217 (1975), 1974.

[25] P. Hilton, G. Mislin, and J. Roitberg. Sphere bundles over spheres and non-cancellation phenomena. pages 34-46. Lecture Notes in Math., Vol. 249, 1971.

[26] M. W. Hirsch. Differential topology. Springer-Verlag, New York, 1976. Graduate Texts in Mathematics, No. 33. 
[27] J. F. P. Hudson. Concordance, isotopy, and diffeotopy. Ann. of Math. (2), 91:425-448, 1970.

[28] B. Jahren and S. Kwasik. Manifolds homotopy equivalent to $\mathbf{R P}^{4} \# \mathbf{R P} \mathbf{P}^{4}$. Math. Proc. Cambridge Philos. Soc., 140(2):245-252, 2006.

[29] B. Jahren and S. Kwasik. How different can $h$-cobordant manifolds be? Bull. Lond. Math. Soc., 47(4):617-630, 2015.

[30] B. Jahren and S. Kwasik. Inertial $h$-cobordisms. arXiv:1711.04546

[31] M. Powell D. Kasprowski, M. Land and P. Teichner. Stable classification of 4-manifolds with 3-manifold fundamental groups. J. Topol., 10(3):827-881, 2017.

[32] M. Kervaire. Le théorème de Barden-Mazur-Stallings. Comment. Math. Helv., 40:31-42, 1965.

[33] M. Kreck. $h$-cobordisms between 1-connected 4-manifolds. Geom. Topol., 5:1-6, 2001.

[34] M. Kreck. Surgery and duality. Ann. of Math. (2), 149(3):707-754, 1999.

[35] S. Kwasik. On low-dimensional s-cobordisms. Comment. Math. Helv., 61(3):415-428, 1986.

[36] S. Kwasik and W. Rosicki. On stability of 3-manifolds. Fund. Math., 182(2):169-180, 2004.

[37] S. Kwasik and W. Rosicki. Cartesian product stabilization of 3-manifolds. Topology Appl., 157(15):2342-2346, 2010.

[38] S. Kwasik and R. Schultz. Vanishing of Whitehead torsion in dimension four. Topology, 31(4):735-756, 1992.

[39] S. Kwasik and R. Schultz. On $h$-cobordisms of spherical space forms. Proc. Amer. Math. Soc., 127(5):1525-1532, 1999.

[40] S. Kwasik and R. Schultz. Toral and exponential stabilization for homotopy spherical space forms. Math. Proc. Camb. Phil. Soc., 137:571-593, 2004

[41] S. Kwasik and R. Schultz. Tangential thickness of manifolds. Proc. Lond. Math. Soc. (3), 110(5):1281-1313, 2015.

[42] M. Latiolais. Simple homotopy type of finite 2-complexes with finite abelian fundamental group. Trans. Amer. Math. Soc., 293(2):655-662, 1986.

[43] T. Lawson. Inertial $h$-cobordisms with finite cyclic fundamental group. Proc. Amer. Math. Soc., 44:492-496, 1974.

[44] T. Lawson. h-cobordisms between simply connected 4-manifolds. Topology Appl., 28(1):75-82, 1988.

[45] W. Ling. Translations on $M \times \mathbf{R}$. In Algebraic and geometric topology (Proc. Sympos. Pure Math., Stanford Univ., Stanford, Calif., 1976), Part 2, Proc. Sympos. Pure Math., XXXII, pages 167-180. Amer. Math. Soc., Providence, R.I., 1978.

[46] W. Lück and H. Reich. The Baum-Connes and the Farrell-Jones conjectures in $K$ - and $L$-theory. In Handbook of K-theory. Vol. 1, 2, pages 703-842. Springer, Berlin, 2005.

[47] B. Mazur. Stable equivalence of differentiable manifolds. Bull. Amer. Math. Soc., 67:377384, 1961.

[48] J. Milnor. Two complexes which are homeomorphic but combinatorially distinct. Ann. of Math. (2), 74:575-590, 1961.

[49] J. Milnor. Lectures on the h-cobordism theorem. Notes by L. Siebenmann and J. Sondow. Princeton University Press, Princeton, N.J., 1965.

[50] J. Milnor. Whitehead torsion. Bull. Amer. Math. Soc., 72:358-426, 1966.

[51] J. Morgan and G. Tian. Ricci flow and the Poincaré conjecture, volume 3 of Clay Mathematics Monographs. American Mathematical Society, Providence, RI; Clay Mathematics Institute, Cambridge, MA, 2007. 
[52] J. Morgan and G. Tian. The geometrization conjecture, volume 5 of Clay Mathematics Monographs. American Mathematical Society, Providence, RI; Clay Mathematics Institute, Cambridge, MA, 2014.

[53] J. Munkres. Obstructions to the smoothing of piecewise-differentiable homeomorphisms. Ann. of Math. (2), 72:521-554, 1960.

[54] C. Rourke and B. Sanderson. Introduction to piecewise-linear topology. Springer Study Edition. Springer-Verlag, Berlin-New York, 1982.

[55] Y. Rudyak. Piecewise linear structures on topological manifolds. World Scientific Publishing Co. Pte. Ltd., Hackensack, NJ, 2016.

[56] S. Smale. Generalized Poincaré's conjecture in dimensions greater than four. Ann. of Math. (2), 74:391-406, 1961.

[57] J. Stallings. On fibering certain 3-manifolds. In Topology of 3-manifolds and related topics (Proc. The Univ. of Georgia Institute, 1961), pages 95-100. Prentice-Hall, Englewood Cliffs, N.J., 1962.

[58] J. Stallings. The piecewise-linear structure of Euclidean space. Proc. Cambridge Philos. Soc., 58:481-488, 1962.

[59] J. Stallings. On infinite processes leading to differentiability in the complement of a point. In Differential and Combinatorial Topology (A Symposium in Honor of Marston Morse), pages 245-254. Princeton Univ. Press, Princeton, N.J., 1965.

[60] R. Torres. Cauchy surfaces and diffeomorphism types of globally hyperbolic spacetimes. Classical Quantum Gravity, 31(17):175006, 8, 2014.

[61] V. G. Turaev. Towards the topological classification of geometric 3-manifolds. In Topology and geometry - Rohlin Seminar, volume 1346 of Lecture Notes in Math., pages 291-323. Springer, Berlin, 1988.

[62] V. G. Turaev. Homeomorphisms of geometric three-dimensional manifolds. Math. Notes, 43(4):307-312, 1988.

[63] L. N. Vaserštern. On the stabilization of the general linear group over a ring. Math. USSR-Sb., 8:383-400, 1969.

[64] C. T. C. Wall. Diffeomorphisms of 4-manifolds. J. London Math. Soc., 39:131-140, 1964.

[65] C. T. C. Wall. On simply-connected 4-manifolds. J. London Math. Soc., 39:141-149, 1964.

[66] C. T. C. Wall. Geometrical connectivity. I. J. London Math. Soc. (2), 3:597-604, 1971.

[67] B. A. F. Wehrfritz. Group and ring theoretic properties of polycyclic groups, volume 10 of Algebra and Applications. Springer-Verlag London, Ltd., London, 2009.

[68] M. Weiss and B. Williams. Automorphisms of manifolds and algebraic $K$-theory. I. K-Theory, 1(6):575-626, 1988.

[69] J. H. C. Whitehead. On $C^{1}$-complexes. Ann. of Math. (2), 41:809-824, 1940.

[70] J. H. C. Whitehead. On the homotopy type of manifolds. Ann. of Math. (2), 41:825-832, 1940.

Jean-Claude HAUSMANN

Department of Mathematics

University of Geneva

CH-1211 Geneva 4, Switzerland

Jean-Claude.hausmann@unige.ch
Bjørn JAHREN

Department of Mathematics

University of Oslo

0316 Oslo, Norway

bjoernj@math.uio.no 